Article

\title{
Q-rung Orthopair Normal Fuzzy Aggregation Operators and Their Application in Multi-Attribute Decision-Making
}

\author{
Zaoli Yang ${ }^{1}$, Xin $\mathrm{Li}^{1}$, Zehong Cao ${ }^{2}$ and Jinqiu $\mathrm{Li}^{3, *}$ \\ 1 College of Economics and Management, Beijing University of Technology, Beijing 100124, China; \\ yangzaoli@bjut.edu.cn (Z.Y.); xinmaer@163.com (X.L.) \\ 2 Discipline of ICT, School of Technology, Environments and Design, College of Sciences and Engineering, \\ University of Tasmania, Hobart, TAS 7001, Australia; zehong.cao@utas.edu.au \\ 3 College of Economics and Management, Harbin Engineering University, Harbin 150001, China \\ * Correspondence: lijinqiu@hrbeu.edu.cn or hanying911@126.com; Tel.: +86-1563-606-6885
}

Received: 6 November 2019; Accepted: 20 November 2019; Published: 22 November 2019

\begin{abstract}
Q-rung orthopair fuzzy set (q-ROFS) is a powerful tool to describe uncertain information in the process of subjective decision-making, but not express vast objective phenomenons that obey normal distribution. For this situation, by combining the q-ROFS with the normal fuzzy number, we proposed a new concept of q-rung orthopair normal fuzzy (q-RONF) set. Firstly, we defined the conception, the operational laws, score function, and accuracy function of q-RONF set. Secondly, we presented some new aggregation operators to aggregate the q-RONF information, including the $\mathrm{q}$-RONF weighted operators, the q-RONF ordered weighted operators, the q-RONF hybrid operator, and the generalized form of these operators. Furthermore, we discussed some desirable properties of the above operators, such as monotonicity, commutativity, and idempotency. Meanwhile, we applied the proposed operators to the multi-attribute decision-making (MADM) problem and established a novel MADM method. Finally, the proposed MADM method was applied in a numerical example on enterprise partner selection, the numerical result showed the proposed method can effectively handle the objective phenomena with obeying normal distribution and complicated fuzzy information, and has high practicality. The results of comparative and sensitive analysis indicated that our proposed method based on q-RONF aggregation operators over existing methods have stronger information aggregation ability, and are more suitable and flexible for MADM problems.
\end{abstract}

Keywords: normal fuzzy number; Q-rung orthopair normal fuzzy sets; q-RONF information aggregation operators; multi-attribute decision-making

\section{Introduction}

On the basis of Zadeh's fuzzy sets [1], Atanassov [2] proposed intuitionistic fuzzy sets (IFS) to characterize the uncertainty according to membership degree (MED) and non-membership degree (NOMED). IFS made up for the insufficiency that Zadeh's fuzzy sets can only characterize fuzzy information by MED. IFS have attracted extensive attention of many scholars who further expanded the IFS, such as interval IFS [3,4], 2-tuple IFS [5], trapezoidal IFS [6,7], intuitionistic normal fuzzy sets [8], intuitionistic uncertain linguistic [9], triangular IFS [10,11], etc. IFS requires that the sum of MED and NOMED is not more than 1 , and this restricts its application in practical decision-making problems. For example, when decision makers (DMs) independently give the MED and NOMED of one attribute of the alternative, the sum of the them may be greater than 1 , and their quadratic sum will be less than or equal to 1 . At the same time, Yager pointed out a situation, with an example [12], when the MED given by the DM is $\sqrt{3} / 2$, and NOMED is $1 / 2$, then $\sqrt{3} / 2+1 / 2>1$, but $(\sqrt{3} / 2)^{2}+(1 / 2)^{2} \leq 1$ 
$(\sqrt{3} / 2)^{2}+(1 / 2)^{2} \leq 1$. For this situation, Yager [12,13] proposed Pythagorean fuzzy sets (PFS), whose characteristic is that the quadratic sum of MED and NOMED is not greater than 1 . Compared with IFS, the PFS characterizes fuzzy information more effectively. Since PFS was proposed, many scholars have conducted in-depth studies [13-23].

Although IFS and PFS have been extensively studied, their expression for fuzzy information is still limited, especially in the extremely complex and contradictory information environment. When the quadratic sum of MED and NOMED given by DMs is still greater than 1, but the sum of three or higher powers is less than 1, IFS and PFS can not deal with such type of information. In order to compensate for the deficiencies of IFS and PFS, and enhance the ability of characterizing fuzzy information. Yager [24] further proposed the concept of q-rung orthopair fuzzy set (q-ROFS) whose characteristics are that the sum of q power of MED and NOMED is bound to 1 , when $q>1$, and that when $q=1$ and 2, q-ROFS becomes IFS and PFS, respectively. Many scholars have carried out extensive research and discussion on the concept of q-ROFS as following three aspects: (1) In terms of basic theory, some studies analyzed some properties of q-rung orthopair fuzzy (q-ROF) functions [25-28], established the distance measure and similarity measure between q-ROFSs [29,30], and defined some new concepts based on q-ROFS [31,32]. (2) In terms of information aggregation operators, some authors established various types of information aggregation operators based on classic operators under q-ROF environment. Such as the family the q-ROF power aggregation operators [33], a series of q-ROF linguistic aggregation operators [34,35], the family of q-ROF Muirhead mean operators [36,37] a sequence of q-ROF Hamy mean operators [38]. In addition, the ROF information aggregation operators based on Archimedean Muirhead mean operators [39], Bonferroni mean operator [40], Heronian mean operator [41-43], and Choquet integral operator [44] were also developed. (3) In terms of the decision-making method, the existing studies combined the q-ROFS with preference relation [45], distance measure [46], and the TOPSIS method $[47,48]$ to develop the corresponding multi-attribute decision-making method.

In real life, a large number of natural and social phenomenon obey normal distribution $[8,49]$, such as "product life span", "stock price", "commodity customer experience evaluation" and so on. In view of these phenomenon, Yang and Ko [49] proposed normal fuzzy number (NFN) to characterize them. Li and Liu [50] pointed out that compared with triangular and trapezoidal fuzzy numbers, NFN has higher derivative continuity, which can characterize natural and social phenomenon more extensively, and their membership functions are closer to human thinking. Li and Liu [50] used examples to prove that the extension of intuitionistic fuzzy number (IFN) based on NFN is better than IFN based on triangular, trapezoidal fuzzy numbers, etc. Therefore, Wang et al. [8] and Wang et al. [51] defined intuitionistic normal fuzzy (INF) number and its operation rules and some information aggregators. Based on these, the concept of interval-valued INF was defined [52], and the family of INF-induced ordered operators [53], and a series of aggregation operators based on classic operators under INF information environment were proposed [54-58].

In summary, PFS and IFS are special cases of q-rung orthopair fuzzy sets. The fuzzy information characterized by q-ROFs is broader and more comprehensive. NIF is closer to human decision-making thinking than triangular and trapezoidal fuzzy numbers. PFS and IFS based on triangular and trapezoidal fuzzy numbers have been reported successively. However, q-rung orthopair fuzzy sets based on INF have not been proposed yet.

In this paper, we defined q-rung orthopair normal fuzzy number, and its operational laws and some aggregation operators, as well as a multi-attribute decision-making method based on q-ROFs algorithm and information aggregator. The rest of this paper is arranged as follows: In Section 2, the basic concepts of NFN and q-ROFs are reviewed. In Section 3, q-RONFN and some of its operational rules are defined. In Section 4, some information aggregation operators in q-RONFs environment are proposed. In Section 5, a multi-attribute decision-making method based on q-RONF Information Aggregation operator is proposed. In Section 6, an example is given to prove the effectiveness of the proposed method. Finally, in Section 7, a conclusion is drawn. 


\section{Preliminaries}

\subsection{The Normal Fuzzy Number}

Definition 1. ([49]) Let $R$ be a real number set, the membership function of

$$
\widetilde{A}(x)=e^{-\left(\frac{x-\alpha}{\sigma}\right)^{2}}(\sigma>0)
$$

is called as a normal fuzzy number (NFN) $\widetilde{A}=(\alpha, \sigma)$, the normal fuzzy number set (NFNS) is denoted by $\widetilde{N}$.

Lemma 1. ([59]) Let $\widetilde{A}, \widetilde{B} \in \widetilde{N}$, denoted by $\widetilde{A}=(\alpha, \sigma), \widetilde{B}=(\beta, \tau)$, then

(1) $\lambda \widetilde{A}=\lambda(\alpha, \sigma)=(\lambda \alpha, \lambda \sigma), \lambda>0$,

(2) $\widetilde{A}+\widetilde{B}=(\alpha, \sigma)+(\beta, \tau)=(\alpha+\beta, \sigma+\tau)$.

Definition 2. ([59]) Let $\widetilde{A}, \widetilde{B} \in \widetilde{N}$, denoted by $\widetilde{A}=(\alpha, \sigma), \widetilde{B}=(\alpha, \sigma)$, then the distance between $\widetilde{A}$ and $\widetilde{B}$ can be defined as

$$
d^{2}(\widetilde{A}, \widetilde{B})=(\alpha-\beta)^{2}+\frac{1}{2}(\sigma-\tau)^{2}
$$

\subsection{The Q-rung Orthopair Fuzzy Number}

Definition 3. ([24]) Let $X$ be an ordinary fixed set, a q-rung orthopair fuzzy set ( $q$-ROFS) $A$ in $X$ defined by $A=\left\{\left\langle x, u_{A}(x), v_{A}(x)\right\rangle \mid x \in X\right\}$, where $u_{A}(x)$ and $v_{A}(x)$ represent the MED and NOMED, respectively, and $u_{A}(x) \in[0,1], v_{A}(x) \in[0,1]$, and $0 \leq u_{A}(x)^{q}+v_{A}(x)^{q} \leq 1 \quad(q \geq 1)$. The degree of indeterminacy is given as $\pi_{A}(x)=\left(u_{A}(x)^{q}+v_{A}(x)^{q}-u_{A}(x)^{q} v_{A}(x)^{q}\right)^{1 / q}$. To simplify the expression, a q-rung orthopair fuzzy number $\left(q\right.$-ROFN) can be denoted as $A=\left(u_{A}, v_{A}\right)$.

Compared with the IFS and PFS, the q-ROFS depicts fuzzy information more broadly. For example, let a fuzzy number be $A=\langle 0.7,0.8\rangle$, the sum of MED and NOMED of $A$ is 1.5 , that exceeds 1 , the quadratic sum of them is 1.13 , also more than 1 , so the IFS and PFS cannot address $A$. However, the sum of 3 power of MED and NOMED is less than 1, hence, we can use q-ROFS to calculate $A$ with making the parameter $q=3$ in q-ROFS.

Definition 4. ([60]) Let $a_{1}=\left(u_{1}, v_{1}\right)$ and $a_{2}=\left(u_{2}, v_{2}\right)$ be two $q$-ROFNs, $\lambda$ be a non-negative real number, then

(1) $a_{1} \oplus a_{2}=\left(\left(u_{1}^{q}+u_{2}^{q}-u_{1}^{q} u_{2}^{q}\right)^{1 / q}, v_{1} v_{2}\right)$,

(2) $a_{1} \otimes a_{2}=\left(u_{1} u_{2},\left(v_{1}^{q}+v_{2}^{q}-v_{1}^{q} v_{2}^{q}\right)^{1 / q}\right)$,

(3) $\lambda a_{1}=\left(\left(1-\left(1-u_{1}^{q}\right)^{\lambda}\right)^{1 / q}, v_{1}^{\lambda}\right)$,

(4) $a_{1}^{\lambda}=\left(u_{1}^{\lambda},\left(1-\left(1-v_{1}^{q}\right)^{\lambda}\right)^{1 / q}\right)$.

Definition 5. ([60]) Let $a=\left(u_{a}, v_{a}\right)$ be a $q$-ROFN, then the score function of $a$ is defined as $S(a)=u_{a}^{q}-v_{a}^{q}$, the accuracy function of $a$ is defined as $H(a)=u_{a}^{q}+v_{a}^{q}$. For any two q-ROFNs, $a_{1}=\left(u_{1}, v_{1}\right)$ and $a_{2}=\left(u_{2}, v_{2}\right)$, then

(1) If $S\left(a_{1}\right)>S\left(a_{2}\right)$, then $a_{1}>a_{2}$; 
(2) If $S\left(a_{1}\right)=S\left(a_{2}\right)$, then

If $H\left(a_{1}\right)>H\left(a_{2}\right)$, then $a_{1}>a_{2}$;

If $H\left(a_{1}\right)=H\left(a_{2}\right)$, then $a_{1}=a_{2}$.

\section{The Q-rung Orthopair Normal Fuzzy Number and Its Operations}

Based on the notions and operations of q-ROFN and NFN, we presented the q-rung orthopair normal fuzzy number (q-RONFN) and its operations.

Definition 6. Let $X$ be an ordinary fixed non-empty set and $(\alpha, \sigma) \in \widetilde{N}, A=\left\langle(\alpha, \sigma),\left(u_{a}, v_{a}\right)\right\rangle$ is a q-rung orthopair normal fuzzy set ( $q$-RONFS), when its membership function is defined as

$$
u_{A}(x)=u_{A} e^{-\left(\frac{x-\alpha}{\sigma}\right)^{2}}, \quad x \in X
$$

and non-membership function is defined as

$$
v_{A}(x)=1-\left(1-v_{A}\right) e^{-\left(\frac{x-\alpha}{\sigma}\right)^{2}}, x \in X,
$$

where $0 \leq u_{A}(x), v_{A}(x) \leq 1,0 \leq\left(u_{A}(x)\right)^{q}+\left(v_{A}(x)\right)^{q} \leq 1,(q \geq 1)$. When $u_{A}=1$ and $v_{A}=0$, the $q$-RONFS will be transformed into a normal fuzzy number. To simplify the expression, a q-rung orthopair normal fuzzy number $(q-R O N F N)$ is denoted as $A=\left\langle(\alpha, \sigma),\left(u_{A}, v_{A}\right)\right\rangle$.

Definition 7. Let $a_{1}=\left\langle\left(\alpha_{1}, \sigma_{1}\right),\left(u_{1}, v_{1}\right)\right\rangle$ and $a_{2}=\left\langle\left(\alpha_{2}, \sigma_{2}\right),\left(u_{2}, v_{2}\right)\right\rangle$ be any two $q-R O N F N s$, and $\lambda$ be $a$ nonnegative real number, then

(1) $a_{1} \oplus a_{2}=\left(\left(\alpha_{1}+\alpha_{2}, \sigma_{1}+\sigma_{2}\right),\left(u_{1}^{q}+u_{2}^{q}-u_{1}^{q} u_{2}^{q}\right)^{1 / q}, v_{1} v_{2}\right)$,

(2) $a_{1} \otimes a_{2}=\left(\left(\alpha_{1} \alpha_{2}, \sigma_{1} \sigma_{2}\right), u_{1} u_{2},\left(v_{1}^{q}+v_{2}^{q}-v_{1}^{q} v_{2}^{q}\right)^{1 / q}\right)$,

(3) $\lambda a_{1}=\left(\left(\lambda \alpha_{1}, \lambda \sigma_{1}\right),\left(1-\left(1-u_{1}^{q}\right)^{\lambda}\right)^{1 / q}, v_{1}^{\lambda}\right)$,

(4) $\quad a_{1}^{\lambda}=\left(\left(\alpha_{1}^{\lambda}, \sigma_{1}^{\lambda}\right), u_{1}^{\lambda},\left(1-\left(1-v_{1}^{q}\right)^{\lambda}\right)^{1 / q}\right)$.

Proposition 1. Let $a_{1}=\left\langle\left(\alpha_{1}, \sigma_{1}\right),\left(u_{1}, v_{1}\right)\right\rangle, a_{2}=\left\langle\left(\alpha_{2}, \sigma_{2}\right),\left(u_{2}, v_{2}\right)\right\rangle$ and $a_{3}=\left\langle\left(\alpha_{3}, \sigma_{3}\right),\left(u_{3}, v_{3}\right)\right\rangle$ be any three $q$-RONFNs, and $\lambda, \lambda_{1}, \lambda_{2}$ be nonnegative real numbers, we can obtain that

(1) $a_{1}+a_{2}=a_{2}+a_{1}$,

(2) $\left(a_{1}+a_{2}\right)+a_{3}=a_{1}+\left(a_{2}+a_{3}\right)$,

(3) $a_{1} \times a_{2}=a_{2} \times a_{1}$,

(4) $\left(a_{1} \times a_{2}\right) \times a_{3}=a_{1} \times\left(a_{2} \times a_{3}\right)$,

(5) $\lambda_{1} a_{1}+\lambda_{2} a_{1}=\left(\lambda_{1}+\lambda_{2}\right) a_{1}$,

(6) $\lambda\left(a_{1}+a_{2}\right)=\lambda a_{1}+\lambda a_{2}$,

(7) $\left(a_{1}^{\lambda_{1}}\right)^{\lambda_{2}}=a_{1}^{\lambda_{1} \lambda_{2}}$.

Proof. According to the Definition 7, we can easily infer that (1), (3), (5), (6) and (7) are obviously right, (2) and (4) need be proved as follows:

For $(2)\left(a_{1}+a_{2}\right)+a_{3}=a_{1}+\left(a_{2}+a_{3}\right)$.

Let the NFN of q-RONFN $r$ be $\widetilde{N}_{r}$, the MED of $\left(a_{1}+a_{2}\right)+a_{3}$ and $a_{1}+\left(a_{2}+a_{3}\right)$ be $u_{\left(a_{1}+a_{2}\right)+a_{3}}$ and $u_{a_{1}+\left(a_{2}+a_{3}\right)}$, and the NOMED of $\left(a_{1}+a_{2}\right)+a_{3}$ and $a_{1}+\left(a_{2}+a_{3}\right)$ be $v_{\left(a_{1}+a_{2}\right)+a_{3}}$ and $v_{a_{1}+\left(a_{2}+a_{3}\right)}$, respectively, and we can obtain that

$$
\widetilde{N}_{\left(r_{1}+r_{2}\right)+r_{3}}=\widetilde{N}_{r_{1}+\left(r_{2}+r_{3}\right)}=\left(\alpha_{1}+\alpha_{2}+\alpha_{3}, \sigma_{1}+\sigma_{2}+\sigma_{3}\right),
$$




$$
\begin{gathered}
u_{\left(a_{1}+a_{2}\right)+a_{3}}=\left(u_{1}^{q}+u_{2}^{q}-u_{1}^{q} u_{2}^{q}+u_{3}^{q}-\left(u_{1}^{q}+u_{2}^{q}-u_{1}^{q} u_{2}^{q}\right) u_{3}^{q}\right)^{1 / q} \\
=\left(u_{1}^{q}+u_{2}^{q}+u_{3}^{q}-u_{1}^{q} u_{2}^{q}-u_{1}^{q} u_{3}^{q}-u_{2}^{q} u_{3}^{q}+u_{1}^{q} u_{2}^{q} u_{3}^{q}\right)^{1 / q} \\
u_{a_{1}+\left(a_{2}+a_{3}\right)}=\left(u_{2}^{q}+u_{3}^{q}-u_{2}^{q} u_{3}^{q}+u_{1}^{q}-\left(u_{2}^{q}+u_{3}^{q}-u_{2}^{q} u_{3}^{q}\right) u_{1}^{q}\right)^{1 / q} \\
=\left(u_{1}^{q}+u_{2}^{q}+u_{3}^{q}-u_{1}^{q} u_{2}^{q}-u_{1}^{q} u_{3}^{q}-u_{2}^{q} u_{3}^{q}+u_{1}^{q} u_{2}^{q} u_{3}^{q}\right)^{1 / q} \\
u_{\left(a_{1}+a_{2}\right)+a_{3}}=u_{a_{1}+\left(a_{2}+a_{3}\right)} .
\end{gathered}
$$

Similarly, we can get that $v_{\left(a_{1}+a_{2}\right)+a_{3}}=v_{a_{1}+\left(a_{2}+a_{3}\right)}$.

Therefore, $\left(a_{1}+a_{2}\right)+a_{3}=a_{1}+\left(a_{2}+a_{3}\right)$.

For (4) $\left(a_{1} \times a_{2}\right) \times a_{3}=a_{1} \times\left(a_{2} \times a_{3}\right)$.

Let the normal fuzzy number of q-RONFNs $r$ be $\widetilde{N}_{r}$, the MED of $\left(a_{1} \times a_{2}\right) \times a_{3}$ and $a_{1} \times\left(a_{2} \times a_{3}\right)$ be $u_{\left(a_{1} \times a_{2}\right) \times a_{3}}$ and $u_{a_{1} \times\left(a_{2} \times a_{3}\right)}$, and the NOMED of $\left(a_{1} \times a_{2}\right) \times a_{3}$ and $a_{1} \times\left(a_{2} \times a_{3}\right)$ be $v_{\left(a_{1} \times a_{2}\right) \times a_{3}}$ and $v_{a_{1} \times\left(a_{2} \times a_{3}\right)}$, respectively, and we can obtain that

$$
\begin{gathered}
\widetilde{N}_{\left(r_{1} \times r_{2}\right) \times r_{3}}=\widetilde{N}_{r_{1} \times\left(r_{2} \times r_{3}\right)}=\left(\alpha_{1} \times \alpha_{2} \times \alpha_{3}, \sigma_{1} \times \sigma_{2} \times \sigma_{3}\right)_{\prime \prime} \\
v_{\left(a_{1} \times a_{2}\right) \times a_{3}}=\left(v_{1}^{q}+v_{2}^{q}-v_{1}^{q} v_{2}^{q}+v_{3}^{q}-\left(v_{1}^{q}+v_{2}^{q}-v_{1}^{q} v_{2}^{q}\right) v_{3}^{q}\right) \\
=\left(v_{1}^{q}+v_{2}^{q}+v_{3}^{q}-v_{1}^{q} v_{2}^{q}-v_{1}^{q} v_{3}^{q}-v_{2}^{q} v_{3}^{q}+v_{1}^{q} v_{2}^{q} v_{3}^{q}\right) \\
v_{\left(a_{1} \times a_{2}\right) \times a_{3}}=\left(v_{2}^{q}+v_{3}^{q}-v_{2}^{q} v_{3}^{q}+v_{1}^{q}-\left(v_{2}^{q}+v_{3}^{q}-v_{2}^{q} v_{3}^{q}\right) v_{1}^{q}\right) \\
=\left(v_{1}^{q}+v_{2}^{q}+v_{3}^{q}-v_{1}^{q} v_{2}^{q}-v_{1}^{q} v_{3}^{q}-v_{2}^{q} v_{3}^{q}+v_{1}^{q} v_{2}^{q} v_{3}^{q}\right) \\
v_{\left(a_{1} \times a_{2}\right) \times a_{3}}=v_{a_{1} \times\left(a_{2} \times a_{3}\right) .}
\end{gathered}
$$

Similarly, we can get that $u_{\left(a_{1} \times a_{2}\right) \times a_{3}}=u_{a_{1} \times\left(a_{2} \times a_{3}\right)}$.

Definition 8. Let $a=\langle(\alpha, \sigma),(u, v)\rangle$ be a $q$-RONFN, its score function is defined as

$$
S_{1}(a)=\alpha\left(u_{a}^{q}-v_{a}^{q}\right), S_{2}(a)=\sigma\left(u_{a}^{q}-v_{a}^{q}\right),
$$

its accuracy function is defined as

$$
H_{1}(a)=\alpha\left(u_{a}^{q}+v_{a}^{q}\right), H_{2}(a)=\sigma\left(u_{a}^{q}+v_{a}^{q}\right) .
$$

Definition 9. Let $a_{1}=\left\langle\left(\alpha_{1}, \sigma_{1}\right),\left(u_{1}, v_{1}\right)\right\rangle, a_{2}=\left\langle\left(\alpha_{2}, \sigma_{2}\right),\left(u_{2}, v_{2}\right)\right\rangle$ be any two $q$-RONFNs, their score functions are $S_{1}(a), S_{2}(a)$, their accuracy functions are $H_{1}(a), H_{2}(a)$, respectively, then we can get

(1) If $S_{1}\left(a_{1}\right)>S_{1}\left(a_{2}\right)$, then $a_{1}>a_{2}$,

(2) If $S_{1}\left(a_{1}\right)=S_{1}\left(a_{2}\right)$ and $H_{1}\left(a_{1}\right)>H_{1}\left(a_{2}\right)$, then $a_{1}>a_{2}$,

(3) If $S_{1}\left(a_{1}\right)=S_{1}\left(a_{2}\right)$ and $H_{1}\left(a_{1}\right)=H_{1}\left(a_{2}\right)$,

If $S_{2}\left(a_{1}\right)<S_{2}\left(a_{2}\right)$, then $a_{1}>a_{2}$,

If $S_{2}\left(a_{1}\right)=S_{2}\left(a_{2}\right)$ and $H_{2}\left(a_{1}\right)<H_{2}\left(a_{2}\right)$, then $a_{1}>a_{2}$,

If $S_{2}\left(a_{1}\right)=S_{2}\left(a_{2}\right)$ and $H_{2}\left(a_{1}\right)=H_{2}\left(a_{2}\right)$, then $a_{1}=a_{2}$.

\section{Q-Rung Orthopair Normal Fuzzy Aggregation Operators}

Based on operational rules of q-RONFN, we will present some q-Rung orthopair normal fuzzy ( $\mathrm{q}-\mathrm{RONF}$ ) aggregation operators.

\subsection{The q-RONF Weighted Aggregation Operators}

Based on the operational rules of q-RONFN and Xu's IFWA [61], the q-RONF weighted averaging and geometry aggregation operators are presented as follows: 
Definition 10. Let $a_{i}=\left\langle\left(\alpha_{i}, \sigma_{i}\right),\left(u_{i}, v_{i}\right)\right\rangle(i=1,2, \cdots, n)$ be a collection of the $q$-RONFNs, and $w=$ $\left(w_{1}, w_{2}, \cdots, w_{n}\right)^{T}$ be the weight vector of $a_{i}$, and $0 \leq w_{i} \leq 1 \quad(i=1,2, \cdots, n)^{T}, \sum_{i=1}^{n} w_{i}=1$, then

$$
\mathrm{q}-\operatorname{RONFWA}\left(a_{1}, a_{2}, \cdots, a_{n}\right)=\sum_{i=1}^{n} w_{i} a_{i}
$$

is called an $q$-RONF weighted averaging ( $q$-RONFWA) operator.

Theorem 1. Let $a_{i}=\left\langle\left(\alpha_{i}, \sigma_{i}\right),\left(u_{i}, v_{i}\right)\right\rangle(i=1,2, \cdots, n)$ be a collection of the $q$-RONFNs, the value by using Definition 10 is still a $q-R O N F N$, that is

$$
\mathrm{q}-\operatorname{RONFWA}\left(a_{1}, a_{2}, \cdots, a_{n}\right)=\left\langle\left(\sum_{i=1}^{n} w_{i} \alpha_{i}, \sum_{i=1}^{n} w_{i} \sigma_{i}\right),\left(\left(1-\prod_{i=1}^{n}\left(1-u_{i}^{q}\right)^{w_{i}}\right)^{1 / q}, \prod_{i=1}^{n} v_{i}^{w_{i}}\right)\right\rangle .
$$

Proof. The mathematical induction method is used to prove the Theorem 1 as follows:

(1) When $n=2$,

Since

$$
w_{1} a_{1}=\left\langle\left(w_{1} \alpha_{1}, w_{1} \sigma_{1}\right),\left(\left(1-\left(1-u_{1}^{q}\right)^{w_{1}}\right)^{1 / q}, v_{1}^{w_{1}}\right)\right\rangle,
$$

and

$$
w_{2} a_{2}=\left\langle\left(w_{2} \alpha_{2}, w_{2} \sigma_{2}\right),\left(\left(1-\left(1-u_{2}^{q}\right)^{w_{2}}\right)^{1 / q}, v_{2}^{w_{2}}\right)\right\rangle,
$$

then

$$
\begin{gathered}
\mathrm{q}-\operatorname{RONFWA}\left(a_{1}, a_{2}\right)=w_{1} \alpha_{1} \oplus w_{2} \alpha_{2} \\
\left(w_{1} \alpha_{1}+w_{2} \alpha_{2}, w_{1} \sigma_{1}+w_{2} \sigma_{2}\right), \\
=\left\langle\left(\left(\begin{array}{c}
\left.\left(1-\left(1-u_{1}^{q}\right)^{w_{1}}\right)^{1 / q}\right)^{q}+\left(\left(1-\left(1-u_{2}^{q}\right)^{w_{2}}\right)^{1 / q}\right)^{q} \\
\left.-\left(\left(1-\left(1-u_{1}^{q}\right)^{q}\right)^{w_{1}}\right)^{1 / q}\right)^{q}\left(\left(1-\left(1-u_{2}^{q}\right)^{w_{2}}\right)^{1 / q}\right)^{q}
\end{array}\right)^{\left.v_{1}^{w_{1}} v_{2}^{w_{2}}\right)}\right)\right. \\
=\left\langle\left(\sum_{i=1}^{2} w_{i} \alpha_{i}, \sum_{i=1}^{2} w_{i} \sigma_{i}\right),\right. \\
\left.=\left\langle\left(\left(1-\left(1-u_{1}^{q}\right)^{w_{1}}\right)+\left(1-\left(1-u_{2}^{q}\right)^{w_{2}}\right)-\left(1-\left(1-u_{1}^{q}\right)^{w_{1}}\right)\left(1-\left(1-u_{2}^{q}\right)^{w_{2}}\right)\right)^{1 / q}, \prod_{i=1}^{2} v_{i}^{w_{i}}\right)\right\rangle \\
\left.=\left\langle\sum_{i=1}^{2} w_{i} \alpha_{i}, \sum_{i=1}^{2} w_{i} \sigma_{i}\right),\left(\left(1-\sum_{i=1}^{2}\left(1-u_{i}^{q}\right)^{w_{i}}\right)^{1 / q}, \prod_{i=1}^{2} v_{i}^{w_{i}}\right)\right\rangle
\end{gathered}
$$

(2) Supposing $n=k, k>2$, that is

$$
\mathrm{q}-\operatorname{RONFWA}\left(a_{1}, a_{2}, \cdots, a_{k}\right)=\left\langle\left(\sum_{i=1}^{k} w_{i} \alpha_{i}, \sum_{i=1}^{k} w_{i} \sigma_{i}\right),\left(\left(1-\prod_{i=1}^{k}\left(1-u_{i}^{q}\right)^{w_{i}}\right)^{1 / q}, \prod_{i=1}^{k} v_{i}^{w_{i}}\right)\right\rangle .
$$


If $n=k+1$, according to the operational laws of q-RONFN, we can get

$$
\begin{aligned}
& \mathrm{q}-\operatorname{RONFWA}\left(a_{1}, a_{2}, \cdots, a_{k}, a_{k+1}\right)=\mathrm{q}-\operatorname{RONFWA}\left(a_{1}, a_{2}, \cdots, a_{k}\right) \oplus w_{k+1} \alpha_{k+1} \\
& \left(\sum_{i=1}^{k} w_{i} \alpha_{i}+w_{k+1} \alpha_{k+1}, \sum_{i=1}^{k} w_{i} \sigma_{i}+w_{k+1} \sigma_{k+1}\right), \\
& =\left\langle\left(\left(\begin{array}{c}
\left.\left(\left(1-\prod_{i=1}^{k}\left(1-u_{i}^{q}\right)^{w_{i}}\right)^{1 / q}\right)^{q}+\left(\left(1-\left(1-u_{k+1}^{q}\right)^{w_{k+1}}\right)^{1 / q}\right)^{q}\right)^{1 / q} \\
-\left(\left(1-\prod_{i=1}^{k}\left(1-u_{i}^{q}\right)^{w_{i}}\right)^{1 / q}\right)^{q}\left(\left(1-\left(1-u_{k+1}^{q}\right)^{w_{k+1}}\right)^{1 / q}\right)^{q} v_{i} v_{k+1}^{w_{k+1}}
\end{array}\right)\right\rangle\right. \\
& \left(\sum_{i=1}^{k+1} w_{i} \alpha_{i}, \sum_{i=1}^{k+1} w_{i} \sigma_{i}\right) \\
& =\left\langle\left(\left(1-\prod_{i=1}^{k}\left(1-u_{i}^{q}\right)^{w_{i}}+1-\left(1-u_{k+1}^{q}\right)^{w_{k+1}}-\left(1-\prod_{i=1}^{k}\left(1-u_{i}^{q}\right)^{w_{i}}\right)\left(1-\left(1-u_{k+1}^{q}\right)^{w_{k+1}}\right)\right)^{1 / q},\right)\right\rangle \\
& =\left\langle\left(\sum_{i=1}^{k+1} w_{i} \alpha_{i}, \sum_{i=1}^{k+1} w_{i} \sigma_{i}\right),\left(\left(1-\prod_{i=1}^{k+1}\left(1-u_{i}^{q}\right)^{w_{i}}\right)^{1 / q}, \prod_{i=1}^{k+1} v_{i}^{w_{i}}\right)\right\rangle
\end{aligned}
$$

(3) According to steps (1) and (2), we can get Theorem 1 holds for any $k$. $\square$

There are some properties can be easily proven as follows:

Theorem 2. (Idempotency). Let $a_{i}=\left\langle\left(\alpha_{i}, \sigma_{i}\right),\left(u_{i}, v_{i}\right)\right\rangle(i=1,2, \cdots, n)$ be a collection of the $q$-RONFNs, if $a_{i}=\left\langle\left(\alpha_{i}, \sigma_{i}\right),\left(u_{i}, v_{i}\right)\right\rangle$ are equal with $a_{i}=a(i=1,2, \cdots, n)$, then

$$
\mathrm{q}-\operatorname{RONFWA}\left(a_{1}, a_{2}, \cdots, a_{n}\right)=a \text {. }
$$

Proof. The process of proof is the same with Theorem 1.

Theorem 3. (Boundedness). Let $a_{i}=\left\langle\left(\alpha_{i}, \sigma_{i}\right),\left(u_{i}, v_{i}\right)\right\rangle(i=1,2, \cdots, n)$ be a collection of the $q$-RONFNs.

If

$$
a^{+}=\left\langle\left(\max _{1 \leq i \leq n}\left\{\alpha_{i}\right\}, \min _{1 \leq i \leq n}\left\{\sigma_{i}\right\}\right),\left(\max _{1 \leq i \leq n}\left\{u_{i}\right\}, \min _{1 \leq i \leq n}\left\{v_{i}\right\}\right)\right\rangle,
$$

and

$$
a^{-}=\left\langle\left(\min _{1 \leq i \leq n}\left\{\alpha_{i}\right\}, \max _{1 \leq i \leq n}\left\{\sigma_{i}\right\}\right),\left(\min _{1 \leq i \leq n}\left\{u_{i}\right\}, \max _{1 \leq i \leq n}\left\{v_{i}\right\}\right)\right\rangle .
$$

Then we have

$$
a^{-} \leq \mathrm{q}-\operatorname{ROFNWA}\left(a_{1}, a_{2}, \cdots, a_{n}\right) \leq a^{+} .
$$

Proof. (1). The normal fuzzy number of $\mathrm{q}-\operatorname{RONFWA}\left(a_{1}, a_{2}, \cdots, a_{n}\right)$, we get

$$
\begin{gathered}
\sum_{i=1}^{n} w_{i} \min _{1 \leq i \leq n}\left\{\alpha_{i}\right\} \leq \sum_{i=1}^{n} w_{i} \alpha_{i j} \leq \sum_{i=1}^{n} w_{i} \max _{1 \leq i \leq n}\left\{\alpha_{i}\right\} \\
\Rightarrow \min _{1 \leq i \leq n}\left\{\alpha_{i}\right\} \sum_{i=1}^{n} w_{i} \leq \sum_{i=1}^{n} w_{i} \alpha_{i j} \leq \max _{1 \leq i \leq n}\left\{\alpha_{i}\right\} \sum_{i=1}^{n} w_{i} \\
\Rightarrow \min _{1 \leq i \leq n}\left\{\alpha_{i}\right\} \leq \sum_{i=1}^{n} w_{i} \alpha_{i j} \leq \max _{1 \leq i \leq n}\left\{\alpha_{i}\right\}
\end{gathered}
$$


and

$$
\begin{gathered}
\sum_{i=1}^{n} w_{i} \min _{1 \leq i \leq n}\left\{\sigma_{i}\right\} \leq \sum_{i=1}^{n} w_{i} \sigma_{i j} \leq \sum_{i=1}^{n} w_{i} \max _{1 \leq i \leq n}\left\{\sigma_{i}\right\} \\
\Rightarrow \min _{1 \leq i \leq n}\left\{\sigma_{i}\right\} \sum_{i=1}^{n} w_{i} \leq \sum_{i=1}^{n} w_{i} \sigma_{i j} \leq \max _{1 \leq i \leq n}\left\{\sigma_{i}\right\} \sum_{i=1}^{n} w_{i} \\
\Rightarrow \min _{1 \leq i \leq n}\left\{\sigma_{i}\right\} \leq \sum_{i=1}^{n} w_{i} \sigma_{i j} \leq \max _{1 \leq i \leq n}\left\{\sigma_{i}\right\}
\end{gathered}
$$

(2). For the MED of $q-\operatorname{RONFWA}\left(a_{1}, a_{2}, \cdots, a_{n}\right)$, we get

$$
\begin{gathered}
\left(1-\prod_{i=1}^{n}\left(1-\min _{1 \leq i \leq n} u_{i}^{q}\right)^{w_{i}}\right)^{1 / q} \leq\left(1-\prod_{i=1}^{k+1}\left(1-u_{i}^{q}\right)^{w_{i}}\right)^{1 / q} \leq\left(1-\prod_{i=1}^{n}\left(1-\max _{1 \leq i \leq n} u_{i}^{q}\right)^{w_{i}}\right)^{1 / q} \\
\Rightarrow\left(1-\left(1-\min _{1 \leq i \leq n} u_{i}^{q}\right)^{\sum_{i=1}^{n} w_{i}}\right)^{1 / q} \leq\left(1-\prod_{i=1}^{n}\left(1-u_{i}^{q}\right)^{w_{i}}\right)^{1 / q} \leq\left(1-\left(1-\max _{1 \leq i \leq n} u_{i}^{q}\right)^{\sum_{i=1}^{n} w_{i}}\right)^{1 / q} \\
\Rightarrow \min _{1 \leq i \leq n}\left\{u_{i}\right\} \leq\left(1-\prod_{i=1}^{n}\left(1-u_{i}^{q}\right)^{w_{i}}\right)^{1 / q} \leq \max _{1 \leq i \leq n}\left\{u_{i}\right\}
\end{gathered}
$$

For the NOMED of $\mathrm{q}-\operatorname{RONFWA}\left(a_{1}, a_{2}, \cdots, a_{n}\right)$, we get

$$
\begin{gathered}
\prod_{i=1}^{n} \min _{1 \leq i \leq n} v_{i}^{w_{i}} \leq \prod_{i=1}^{n} v_{i}^{w_{i}} \leq \prod_{i=1}^{n} \max _{1 \leq i \leq n} v_{i}^{w_{i}} \\
\Rightarrow \min _{1 \leq i \leq n} v_{i}^{\sum_{i=1}^{n} w_{i}} \leq \prod_{i=1}^{n} v_{i}^{w_{i}} \leq \max _{1 \leq i \leq n} v_{i}^{\sum_{i=1}^{n}} w_{i} \\
\Rightarrow \min _{1 \leq i \leq n}\left\{v_{i}\right\} \leq \prod_{i=1}^{n} v_{i}^{w_{i}} \leq \max _{1 \leq i \leq n}\left\{v_{i}\right\}
\end{gathered}
$$

Then, we have

$$
\begin{gathered}
\min _{1 \leq i \leq n}\left\{\alpha_{i}\right\}\left(\min _{1 \leq i \leq n}\left\{u_{i}\right\}-\max _{1 \leq i \leq n}\left\{v_{i}\right\}\right) \leq \alpha\left(\left(1-\prod_{i=1}^{n}\left(1-u_{i}^{q}\right)^{w_{i}}\right)^{1 / q}-\prod_{i=1}^{n} v_{i}^{w_{i}}\right) \\
\leq \max _{1 \leq i \leq n}\left\{\alpha_{i}\right\}\left(\max _{1 \leq i \leq n}\left\{u_{i}\right\}-\min _{1 \leq i \leq n}\left\{v_{i}\right\}\right)
\end{gathered}
$$

and

$$
\Rightarrow S_{1}\left(a^{-}\right) \leq S_{1}(a) \leq S_{1}\left(a^{+}\right) .
$$

Based on steps (1)-(3) and Definition 7, we have $a^{-} \leq \mathrm{q}-\operatorname{RONFWA}\left(a_{1}, a_{2}, \cdots, a_{n}\right) \leq a^{+}$.

Theorem 4. (Monotonicity). Let $a_{i}=\left\langle\left(\alpha_{i}, \sigma_{i}\right),\left(u_{i}, v_{i}\right)\right\rangle$ and $\widetilde{a}_{i}=\left\langle\left(\widetilde{\alpha}_{i}, \widetilde{\sigma}_{i}\right),\left(\widetilde{u}_{i}, \widetilde{v}_{i}\right)\right\rangle(i=1,2, \cdots, n)$ be any two sets of the $q$-RONFN, if $\alpha_{i} \geq \widetilde{\alpha}_{i}, \sigma_{i} \leq \widetilde{\sigma}_{i}, u_{i} \geq \widetilde{u}_{i}, v_{i} \leq \widetilde{v}_{i}$ for all $i$, then

$$
\mathrm{q}-\operatorname{RONFWA}\left(a_{1}, a_{2}, \cdots, a_{n}\right) \geq \mathrm{q}-\operatorname{RONFWA}\left(\widetilde{a}_{1}, \tilde{a}_{2}, \cdots, \widetilde{a}_{n}\right)
$$

Proof. The process of proof is the same with Theorem 2.

Definition 11. Let $a_{i}=\left\langle\left(\alpha_{i}, \sigma_{i}\right),\left(u_{i}, v_{i}\right)\right\rangle(i=1,2, \cdots, n)$ be a collection of the $q$-RONFN, and $w=$ $\left(w_{1}, w_{2}, \cdots, w_{n}\right)^{T}$ be the weight vector of $a_{i}$, and $0 \leq w_{i} \leq 1(i=1,2, \cdots, n)^{T}, \sum_{i=1}^{n} w_{i}=1$, then

$$
\mathrm{q}-\operatorname{RONFWG}\left(a_{1}, a_{2}, \cdots, a_{n}\right)=\prod_{i=1}^{n}\left(a_{i}\right)^{w_{i}}
$$

is called an q-RONF weighted geometry ( $q$-RONFWG) operator. 
Theorem 5. Let $a_{i}=\left\langle\left(\alpha_{i}, \sigma_{i}\right),\left(u_{i}, v_{i}\right)\right\rangle(i=1,2, \cdots, n)$ be a collection of the $q$-RONFN, by using Definition 11 , the value is still a $q-R O N F N$, that is

$$
\mathrm{q}-\operatorname{RONFWG}\left(a_{1}, a_{2}, \cdots, a_{n}\right)=\left\langle\left(\prod_{i=1}^{n} \alpha_{i}^{w_{i}}, \prod_{i=1}^{n} \sigma_{i}^{w_{i}}\right),\left(\prod_{i=1}^{n} u_{i}^{w_{i}},\left(1-\prod_{i=1}^{n}\left(1-v_{i}^{q}\right)^{w_{i}}\right)^{1 / q}\right)\right\rangle
$$

Proof. The mathematical induction method is used to prove Theorem 5 , as follows:

(1) When $n=2$,

Since

$$
a_{1}^{w_{1}}=\left\langle\left(\alpha_{1}^{w_{1}}, \sigma_{1}^{w_{1}}\right),\left(u_{1}^{w_{1}},\left(1-\left(1-v_{1}^{q}\right)^{w_{1}}\right)^{1 / q}\right)\right\rangle
$$

and

$$
a_{2}^{w_{2}}=\left\langle\left(\alpha_{2}^{w_{2}}, \sigma_{2}^{w_{2}}\right),\left(u_{2}^{w_{2}},\left(1-\left(1-v_{2}^{q}\right)^{w_{2}}\right)^{1 / q}\right)\right\rangle
$$

then

$$
\begin{gathered}
\mathrm{q}-\operatorname{RONFWG}\left(a_{1}, a_{2}\right)=\alpha_{1}^{w_{1}} \otimes \alpha_{2}^{w_{2}} \\
\left(\alpha_{1}^{w_{1}} \alpha_{2}^{w_{2}}, \sigma_{1}^{w_{1}} \sigma_{2}^{w_{2}}\right), \\
=\left\langle u_{1}^{w_{1}} u_{2}^{w_{2}},\left(\left(\begin{array}{c}
\left.\left(1-\left(1-v_{1}^{q}\right)^{w_{1}}\right)^{1 / q}\right)^{q}+\left(\left(1-\left(1-v_{2}^{q}\right)^{w_{2}}\right)^{1 / q}\right)^{q} \\
-\left(\left(1-\left(1-v_{1}^{q}\right)^{w_{1}}\right)^{1 / q}\right)^{q}\left(\left(1-\left(1-v_{2}^{q}\right)^{w_{2}}\right)^{1 / q}\right)^{q}
\end{array}\right)\right\rangle\right. \\
\left(\prod_{i}^{2} \alpha_{i}^{w_{i}}, \prod_{i=1}^{2} \sigma_{i}^{w_{i}}\right), \\
=\left\langle\left(\prod_{i=1}^{2} u_{i}^{w_{i}},\left(\left(1-\left(1-v_{1}^{q}\right)^{w_{1}}\right)+\left(1-\left(1-v_{2}^{q}\right)^{w_{2}}\right)-\left(1-\left(1-v_{1}^{q}\right)^{w_{1}}\right)\left(1-\left(1-v_{2}^{q}\right)^{w_{2}}\right)\right)^{1 / q}\right)\right\rangle \\
=\left\langle\left(\sum_{i=1}^{2} \alpha_{i}^{w_{i}}, \sum_{i=1}^{2} \sigma_{i}^{w_{i}}\right),\left(\prod_{i=1}^{2} u_{i}^{w_{i}},\left(1-\sum_{i=1}^{2}\left(1-v_{i}^{q}\right)^{w_{i}}\right)^{1 / q}\right)\right\rangle
\end{gathered}
$$

(2) Supposing $n=k, k>2$, that is

$$
\mathrm{q}-\operatorname{RONFWG}\left(a_{1}, a_{2}, \cdots, a_{k}\right)=\left\langle\left(\left(\prod_{i}^{k} \alpha_{i}^{w_{i}}, \prod_{i=1}^{k} \sigma_{i}^{w_{i}}\right)\right),\left(\prod_{i=1}^{k} u_{i}^{w_{i}},\left(1-\sum_{i=1}^{k}\left(1-v_{i}^{q}\right)^{w_{i}}\right)^{1 / q}\right)\right\rangle .
$$

If $n=k+1$, according to the operational laws of $\mathrm{q}$-RONFN, we can get

$$
\begin{aligned}
& \mathrm{q}-\operatorname{RONFWG}\left(a_{1}, a_{2}, \cdots, a_{k}, a_{k+1}\right)=\mathrm{q}-\operatorname{RONFWG}\left(a_{1}, a_{2}, \cdots, a_{k}\right) \otimes w_{k+1} \alpha_{k+1} \\
& \left(\prod_{i=1}^{k} \alpha_{i}^{w_{i}} \alpha_{k+1}^{w_{k+1}}, \prod_{i=1}^{k} \sigma_{i}^{w_{i}} \sigma_{k+1}^{w_{k+1}}\right), \\
& =\left\langle\left(\prod_{i=1}^{k} u_{i}^{w_{i}} u_{k+1}^{w_{k+1}},\left(\begin{array}{c}
\left(\left(1-\prod_{i=1}^{k}\left(1-v_{i}^{q}\right)^{w_{i}}\right)^{1 / q}\right)^{q}+\left(\left(1-\left(1-v_{k+1}^{q}\right)^{w_{k+1}}\right)^{1 / q}\right)^{q} \\
-\left(\left(1-\prod_{i=1}^{k}\left(1-v_{i}^{q}\right)^{w_{i}}\right)^{1 / q}\right)^{q}\left(\left(1-\left(1-v_{k+1}^{q}\right)^{w_{k+1}}\right)^{1 / q}\right)^{q}
\end{array}\right)^{q / q}\right)\right\rangle \\
& \left(\prod_{i=1}^{k+1} \alpha_{i}^{w_{i}}, \prod_{i=1}^{k+1} \sigma_{i}^{w_{i}}\right) \\
& =\left\langle\left(\prod_{i=1}^{k+1} u_{i}^{w_{i}},\left(1-\prod_{i=1}^{k}\left(1-v_{i}^{q}\right)^{w_{i}}+1-\left(1-v_{k+1}^{q}\right)^{w_{k+1}}-\left(1-\prod_{i=1}^{k}\left(1-v_{i}^{q}\right)^{w_{i}}\right)\left(1-\left(1-v_{k+1}^{q}\right)^{w_{k+1}}\right)\right)^{1 / q}\right)\right\rangle \\
& =\left\langle\left(\prod_{i=1}^{k+1} \alpha_{i}^{w_{i}}, \prod_{i=1}^{k+1} \sigma_{i}^{w_{i}}\right),\left(\prod_{i=1}^{k+1} u_{i}^{w_{i}},\left(1-\prod_{i=1}^{k+1}\left(1-v_{i}^{q}\right)^{w_{i}}\right)^{1 / q}\right)\right\rangle
\end{aligned}
$$


(3) Based on steps (1) and (2), we can get that Theorem 5 holds for any $k$.

According to Theorems 2-4, we can similarly prove the properties of idempotency, monotonicity and boundedness for q-RONFNWG operator.

\subsection{The Q-RONF Ordered Weighted Aggregation Operators}

Considering the ordered position weight of each q-RONFN, according to the ordered weight averaging (OWA) operator [61] and the ordered weighted geometric (OWG) operator [62], some q-RONFNs ordered weighted aggregation operators are presented as follows:

Definition 12. Let $a_{i}=\left\langle\left(\alpha_{i}, \sigma_{i}\right),\left(u_{i}, v_{i}\right)\right\rangle(i=1,2, \cdots, n)$ be a collection of the $q-R O N F N$, and $w_{j}=$ $\left(w_{1}, w_{2}, \cdots, w_{n}\right)^{T}$ be the weight vector of aggregation-associated, and $0 \leq w_{i} \leq 1(i=1,2, \cdots, n)^{T}, \sum_{i=1}^{n} w_{i}=$ $1, a_{\theta(i)}=\left\langle\left(\alpha_{\theta(i)}, \sigma_{\theta(i)}\right),\left(u_{\theta(i)}, v_{\theta(i)}\right)\right\rangle(i=1,2, \cdots, n)$ be the ith largest of them, then

(1) A q-RONF ordered weighted averaging ( $q$-RONFOWA) operator is a mapping $q$-RONFOWA: $a^{n} \rightarrow a$, where

$$
\begin{gathered}
\mathrm{q}-\operatorname{RONFOWA}\left(a_{1}, a_{2}, \cdots, a_{n}\right)=\sum_{i=1}^{n} w_{i} a_{\theta(i)} \\
=\left\langle\left(\sum_{i=1}^{n} w_{i} \alpha_{\theta(i)}, \sum_{i=1}^{n} w_{i} \sigma_{\theta(i)}\right),\left(\left(1-\prod_{i=1}^{n}\left(1-u_{\theta(i)}^{q}\right)^{w_{i}}\right)^{1 / q}, \prod_{i=1}^{n} v_{\theta(i)}^{w_{i}}\right)\right\rangle
\end{gathered}
$$

(2) A q-RONF ordered weighted geometric ( $q$-RONFOWG) operator is a mapping $q$-RONFOWG: $a^{n} \rightarrow a$, where

$$
\mathrm{q}-\operatorname{RONFOWG}\left(a_{1}, a_{2}, \cdots, a_{n}\right)=\left\langle\left(\prod_{i=1}^{n} \alpha_{\theta(i)^{\prime}}^{w_{i}} \prod_{i=1}^{n} \sigma_{\theta(i)}^{w_{i}}\right),\left(\prod_{i=1}^{n} u_{\theta(i)^{\prime}}^{w_{i}}\left(1-\prod_{i=1}^{n}\left(1-v_{\theta(i)}^{q}\right)^{w_{i}}\right)^{1 / q}\right)\right\rangle .
$$

According to Theorems 2 and 3. We can similarly prove the properties of idempotency and boundedness for q-RONFOWA and q-RONFOWG operators. What is more, the q-RONFOWA and q-RONFOWG operators also have the property of commutativity, the proving process for the property of commutativity of q-RONFOWA is showed, as follows:

Theorem 6. (commutativity). Let $a_{i}=\left\langle\left(\alpha_{i}, \sigma_{i}\right),\left(u_{i}, v_{i}\right)\right\rangle(i=1,2, \cdots, n)$ be a collection of the $q$-RONFN, if $\left(\widetilde{a}_{1}, \widetilde{a}_{2}, \cdots, \widetilde{a}_{n}\right)$ is any permutation of $\left(a_{1}, a_{2}, \cdots, a_{n}\right)$, then

$$
\mathrm{q}-\operatorname{RONFOWA}\left(\tilde{a}_{1}, \tilde{a}_{2}, \cdots, \tilde{a}_{n}\right)=\mathrm{q}-\operatorname{RONFOWA}\left(a_{1}, a_{2}, \cdots, a_{n}\right) .
$$

Proof. Since $\left(\widetilde{a}_{1}, \widetilde{a}_{2}, \cdots, \widetilde{a}_{n}\right)$ is any permutation of $\left(a_{1}, a_{2}, \cdots, a_{n}\right)$, let $\widetilde{a}_{\theta(i)}=a_{\theta(i)}(i=1,2, \cdots, n)$ be the $i$ th largest of them, based on the definition 7 , we can get

$$
\mathrm{q}-\operatorname{RONFOWG}\left(\widetilde{a}_{1}, \widetilde{a}_{2}, \cdots, \widetilde{a}_{n}\right)=\mathrm{q}-\operatorname{RONFOWG}\left(a_{1}, a_{2}, \cdots, a_{n}\right) .
$$

\subsection{The Generalized q-RONF Weighted Aggregation Operators}

As generalizations of the q-RONFWA and q-RONFWG operators, some generalized q-rung normal fuzzy weighted aggregation operators are introduced in the following. 
Definition 13. Let $a_{i}=\left\langle\left(\alpha_{i}, \sigma_{i}\right),\left(u_{i}, v_{i}\right)\right\rangle(i=1,2, \cdots, n)$ be a collection of the $q$-RONFN, and $w_{i}=$ $\left(w_{1}, w_{2}, \cdots, w_{n}\right)^{T}$ be the weight vector of $a_{i}$, and $0 \leq w_{i} \leq 1(i=1,2, \cdots, n)^{T}, \sum_{i=1}^{n} w_{i}=1, \lambda$ be a parameter and $\lambda \in(-\infty, 0) \cup(0,+\infty)$ then

$$
\operatorname{Gq}-\operatorname{RONFWA}\left(a_{1}, a_{2}, \cdots, a_{n}\right)=\left(\sum_{i=1}^{n} w_{i} a_{i}^{\lambda}\right)^{1 / \lambda}
$$

is called a generalized q-RONF weighted averaging (Gq-RONFWA) operator.

Theorem 7. Let $a_{i}=\left\langle\left(\alpha_{i}, \sigma_{i}\right),\left(u_{i}, v_{i}\right)\right\rangle(i=1,2, \cdots, n)$ be a collection of the $q$-RONFN, based on the operations of $q$-RONFN, the Gq-RONFWA operator is still a $q$-RONFN, that is

$$
\operatorname{Gq}-\operatorname{RONFWA}\left(a_{1}, a_{2}, \cdots, a_{n}\right)=\left\langle\left(\begin{array}{c}
\left(\left(\sum_{i=1}^{n} w_{i} \alpha_{i}^{\lambda}\right)^{1 / \lambda},\left(\sum_{i=1}^{n} w_{i} \sigma_{i}^{\lambda}\right)^{1 / \lambda}\right) \\
\left(\left(1-\prod_{i=1}^{n}\left(1-u_{i}^{q \lambda}\right)^{w_{i}}\right)^{1 / q}\right)^{1 / \lambda}, \\
\left(1-\left(1-\left(\prod_{i=1}^{n}\left(\left(1-\left(1-v_{i}^{q}\right)^{\lambda}\right)^{1 / q}\right)^{w_{i}}\right)^{q}\right)^{1 / \lambda}\right)^{1 / q}
\end{array}\right)\right\rangle
$$

Proof. The mathematical induction method is used to prove the follow formula firstly:

$$
\sum_{i=1}^{n} w_{i} \alpha_{i}^{\lambda}=\left\langle\left(\left(1-\prod_{i=1}^{n}\left(1-u_{i}^{q \lambda}\right)^{w_{i}}\right)^{1 / q}, \prod_{i=1}^{n}\left(\left(1-\left(1-v_{i}^{q}\right)^{\lambda}\right)^{1 / q}\right)^{w_{i}} \alpha_{i}^{\lambda}, \sum_{i=1}^{n} w_{i} \sigma_{i}^{\lambda}\right),\right.
$$

(1) When $n=2$,

Since

$$
w_{1} a_{1}^{\lambda}=\left\langle\left(w_{1} \alpha_{1}^{\lambda}, w_{1} \sigma_{1}^{\lambda}\right),\left(\left(1-\left(1-u_{1}^{\lambda q}\right)^{w_{1}}\right)^{1 / q},\left(\left(1-\left(1-v_{1}^{q}\right)^{\lambda}\right)^{1 / q}\right)^{w_{1}}\right)\right\rangle
$$

and

$$
w_{2} a_{2}^{\lambda}=\left\langle\left(w_{2} \alpha_{2}^{\lambda}, w_{2} \sigma_{2}^{\lambda}\right),\left(\left(1-\left(1-u_{2}^{\lambda q}\right)^{w_{2}}\right)^{1 / q},\left(\left(1-\left(1-v_{2}^{q}\right)^{\lambda}\right)^{1 / q}\right)^{w_{2}}\right)\right\rangle
$$


then

$$
\begin{aligned}
& \sum_{i=1}^{2} w_{i} a_{i}^{\lambda}=w_{1} a_{1}^{\lambda} \oplus w_{2} a_{2}^{\lambda} \\
& =\left\langle\left(w_{1} \alpha_{1}^{\lambda}+w_{2} \alpha_{2}^{\lambda}, w_{1} \sigma_{1}^{\lambda}+w_{2} \sigma_{2}^{\lambda}\right),\left(\begin{array}{c}
\left.\left(\left(1-\left(1-u_{1}^{\lambda q}\right)^{w_{1}}\right)^{1 / q}\right)^{q}+\left(\left(1-\left(1-u_{2}^{\lambda q}\right)^{w_{2}}\right)^{1 / q}\right)^{q}-\right)^{1 / q} \\
\left(\left(1-\left(1-u_{1}^{\lambda q}\right)^{w_{1}}\right)^{1 / q}\right)^{q}\left(\left(1-\left(1-u_{2}^{\lambda q}\right)^{w_{2}}\right)^{1 / q}\right)^{q}
\end{array}\right\rangle\right. \\
& ,\left(\left(1-\left(1-v_{1}^{q}\right)^{\lambda}\right)^{1 / q}\right)^{w_{1}}\left(\left(1-\left(1-v_{2}^{q}\right)^{\lambda}\right)^{1 / q}\right)^{w_{2}}
\end{aligned}
$$

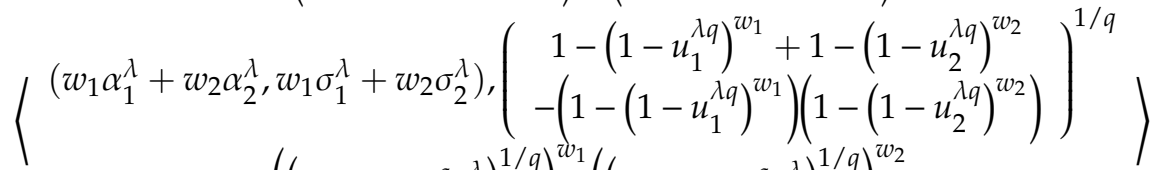

$$
\begin{aligned}
& ,\left(\left(1-\left(1-v_{1}^{q}\right)^{\lambda}\right)^{1 / q}\right)^{w_{1}}\left(\left(1-\left(1-v_{2}^{q}\right)^{\lambda}\right)^{1 / q}\right)^{w_{2}} \\
& =\left\langle\left(\sum_{i=1}^{2} w_{i} \alpha_{i}^{\lambda}, \sum_{i=1}^{2} w_{i} \sigma_{i}^{\lambda}\right),\left(\left(1-\prod_{i=1}^{2}\left(1-u_{i}^{q \lambda}\right)^{w_{i}}\right)^{1 / q}, \prod_{i=1}^{2}\left(\left(1-\left(1-v_{i}^{q}\right)^{\lambda}\right)^{1 / q}\right)^{w_{i}}\right)\right\rangle
\end{aligned}
$$

(2) Supposing $n=k, k>2$, that is

$$
\sum_{i=1}^{k} w_{i} a_{i}^{\lambda}=w_{1} a_{1}^{\lambda} \oplus w_{2} a_{2}^{\lambda} \oplus \cdots \oplus w_{k} a_{k}^{\lambda}=\left\langle\left(\left(1-\prod_{i=1}^{k}\left(1-u_{i}^{q \lambda}\right)^{w_{i}}\right)^{1 / q}, \prod_{i=1}^{k}\left(\left(1-\left(1-v_{i}^{q}\right)^{\lambda}\right)^{1 / q}\right)^{w_{i}}\right)\right\rangle .
$$

If $n=k+1$, according to the operational laws of q-RONFN, we can get

$$
\begin{aligned}
& \sum_{i=1}^{k+1} w_{i} a_{i}^{\lambda}+w_{i} a_{i}^{\lambda}=w_{1} a_{1}^{\lambda} \oplus w_{2} a_{2}^{\lambda} \oplus \cdots \oplus w_{k} a_{k}^{\lambda} \oplus w_{k+1} a_{k+1}^{\lambda} \\
& \left(\sum_{i=1}^{k} w_{i} \alpha_{i}^{\lambda}+w_{k+1} \alpha_{k+1}^{\lambda}, \sum_{i=1}^{k} w_{i} \sigma_{i}^{\lambda}+w_{k+1} \sigma_{k+1}^{\lambda}\right),
\end{aligned}
$$

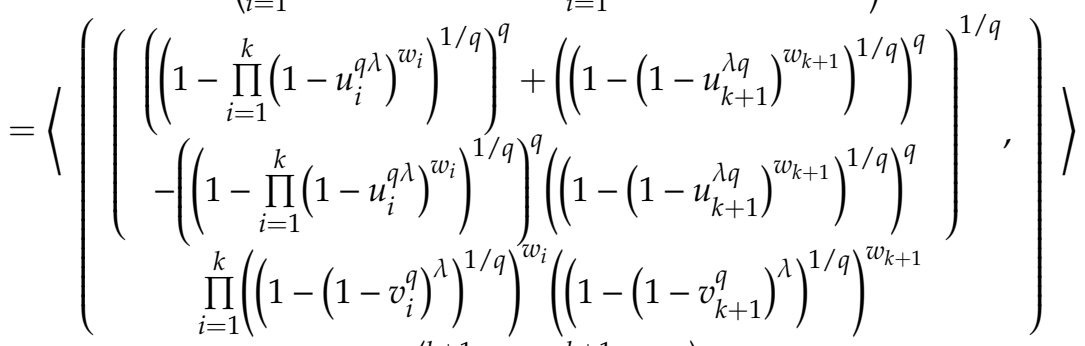

$$
\begin{aligned}
& \left(\sum_{i=1}^{k+1} w_{i} \alpha_{i}^{\lambda}, \sum_{i=1}^{k+1} w_{i} \sigma_{i}^{\lambda}\right) \\
& =\left\langle\left(\left(1-\prod_{i=1}^{k}\left(1-u_{i}^{q \lambda}\right)^{w_{i}}+1-\left(1-u_{k+1}^{\lambda q}\right)^{w_{k+1}}-\left(1-\prod_{i=1}^{k}\left(1-u_{i}^{q \lambda}\right)^{w_{i}}\right)\left(1-\left(1-u_{k+1}^{\lambda q}\right)^{w_{k+1}}\right)\right)^{1 / q},\right)\right\rangle \\
& =\left\langle\left(\sum_{i=1}^{k+1} w_{i} \alpha_{i}^{\lambda}, \sum_{i=1}^{k+1} w_{i} \sigma_{i}^{\lambda}\right),\left(\left(1-\prod_{i=1}^{k+1}\left(1-u_{i}^{q \lambda}\right)^{w_{i}}\right)^{1 / q}, \prod_{i=1}^{k+1}\left(\left(1-\left(1-v_{i}^{q}\right)^{\lambda}\right)^{1 / q}\right)^{w_{i}}\right)\right\rangle
\end{aligned}
$$


then

$$
\left.\left.\left.\left.\left(\sum_{i=1}^{n} w_{i} \alpha_{i}^{\lambda}\right)^{1 / \lambda}=\left\langle\left(\left(\left(1-\prod_{i=1}^{n}\left(1-u_{i}^{q \lambda}\right)^{w_{i}}\right)^{1 / q}\right)^{1 / \lambda},\left(1-\left(1-\left(\prod_{i=1}^{n} w_{i} \alpha_{i}^{\lambda}\right)^{1 / \lambda},\left(\sum_{i=1}^{n} w_{i} \sigma_{i}^{\lambda}\right)^{1 / \lambda}\right),\left(1-v_{i}^{q}\right)^{\lambda}\right)^{1 / q}\right)^{w_{i}}\right)^{q}\right)^{1 / \lambda}\right)^{1 / q}\right)\right\rangle
$$

Of particular note, if $\lambda=1$, the Gq-RONFWA operator is reduced to the q-RONFWA operator. The Gq-RONFWA operator has the properties such as boundedness, and idempotency and monotonicity, which can be exemplified similar to Theorems $2-4$.

Definition 14. Let $a_{i}=\left\langle\left(\alpha_{i}, \sigma_{i}\right),\left(u_{i}, v_{i}\right)\right\rangle(i=1,2, \cdots, n)$ be a collection of the $q$-RONFN, and $w_{i}=$ $\left(w_{1}, w_{2}, \cdots, w_{n}\right)^{T}$ be the weight vector of $a_{i}$, and $0 \leq w_{i} \leq 1(i=1,2, \cdots, n)^{T}, \sum_{i=1}^{n} w_{i}=1, \lambda$ be a parameter and $\lambda \in(-\infty, 0) \cup(0,+\infty)$ then

$$
\operatorname{Gq}-\operatorname{RONFWG}\left(a_{1}, a_{2}, \cdots, a_{n}\right)=\frac{1}{\lambda} \prod_{i=1}^{n}\left(\lambda a_{i}\right)^{w_{i}}
$$

is called a generalized q-RONFWG (Gq-RONFWG) operator.

Theorem 8. Let $a_{i}=\left\langle\left(\alpha_{i}, \sigma_{i}\right),\left(u_{i}, v_{i}\right)\right\rangle(i=1,2, \cdots, n)$ be a collection of the $q$-RONFN, based on the operations of $q-R O N F N$, the Gq-RONFWG operator is still a $q$-RONFN, that is

$$
\operatorname{Gq}-\operatorname{RONFWG}\left(a_{1}, a_{2}, \cdots, a_{n}\right)=\left\langle\left(\begin{array}{c}
\left(\frac{1}{\lambda} \prod_{i=1}^{n}\left(\lambda \alpha_{i}\right)^{w_{i}}, \frac{1}{\lambda} \prod_{i=1}^{n}\left(\lambda \sigma_{i}\right)^{w_{i}}\right), \\
\left(\left(1-\prod_{i=1}^{n}\left(1-v_{i}^{q \lambda}\right)^{w_{i}}\right)^{1 / q}\right)^{1 / \lambda}
\end{array}\right)\right\rangle
$$

Proof. The mathematical induction method is used to prove the following formula firstly:

$$
\prod_{i=1}^{n}\left(\lambda a_{i}\right)^{w_{i}}=\left\langle\left(\prod_{i=1}^{n}\left(\lambda \alpha_{i}\right)^{w_{i}}, \prod_{i=1}^{n}\left(\lambda \sigma_{i}\right)^{w_{i}}\right),\left(\prod_{i=1}^{n}\left(\left(1-\left(1-u_{i}^{q}\right)^{\lambda}\right)^{1 / q}\right)^{w_{i}},\left(1-\prod_{i=1}^{n}\left(1-v_{i}^{q \lambda}\right)^{w_{i}}\right)^{1 / q}\right)\right\rangle .
$$

(1) When $n=2$,

Since

$$
\left(\lambda a_{1}\right)^{w_{1}}=\left\langle\left(\left(\lambda \alpha_{1}\right)^{w_{1}},\left(\lambda \sigma_{1}\right)^{w_{1}}\right),\left(\left(\left(1-\left(1-u_{1}^{q}\right)^{\lambda}\right)^{1 / q}\right)^{w_{1}},\left(1-\left(1-v_{1}^{\lambda q}\right)^{w_{1}}\right)^{1 / q}\right)\right),
$$

and

$$
\left(\lambda a_{2}\right)^{w_{2}}=\left\langle\left(\left(\lambda \alpha_{2}\right)^{w_{2}},\left(\lambda \sigma_{2}\right)^{w_{2}}\right),\left(\left(\left(1-\left(1-u_{2}^{q}\right)^{\lambda}\right)^{1 / q}\right)^{w_{2}},\left(1-\left(1-v_{2}^{\lambda q}\right)^{w_{2}}\right)^{1 / q}\right)\right\rangle .
$$


Then

$$
\begin{gathered}
\prod_{i=1}^{2}\left(\lambda a_{i}\right)^{w_{i}}=\left(\lambda a_{1}\right)^{w_{1}} \otimes\left(\lambda a_{1}\right)^{w_{1}} \\
\begin{array}{c}
\left(\left(\lambda \alpha_{1}\right)^{w_{1}}\left(\lambda \alpha_{2}\right)^{w_{2}},\left(\lambda \sigma_{1}\right)^{w_{1}}\left(\lambda \sigma_{2}\right)^{w_{2}}\right),\left(\left(1-\left(1-u_{1}^{q}\right)^{\lambda}\right)^{1 / q}\right)^{w_{1}}\left(\left(1-\left(1-u_{2}^{q}\right)^{\lambda}\right)^{1 / q}\right)^{w_{2}}, \\
\left.\left(\left(1-\left(1-v_{2}^{\lambda q}\right)^{w_{2}}\right)^{1 / q}\right)^{q}+\left(\left(1-\left(1-v_{2}^{\lambda q}\right)^{w_{2}}\right)^{1 / q}\right)^{q}-\right)^{1 / q} \\
\left.\left(\left(1-\left(1-v_{2}^{\lambda q}\right)^{w_{2}}\right)^{1 / q}\right)^{q}\left(\left(\left(1-\left(1-v_{2}^{\lambda q}\right)^{w_{2}}\right)^{1 / q}\right)^{q}\right)^{q}\right)^{q}
\end{array} \\
\left(\begin{array}{c}
\left.\left(\lambda \alpha_{1}\right)^{w_{1}}\left(\lambda \alpha_{2}\right)^{w_{2}},\left(\lambda \sigma_{1}\right)^{w_{1}}\left(\lambda \sigma_{2}\right)^{w_{2}}\right),\left(\left(1-\left(1-u_{1}^{q}\right)^{\lambda}\right)^{1 / q}\right)^{w_{1}}\left(\left(1-\left(1-u_{2}^{q}\right)^{\lambda}\right)^{1 / q}\right)^{w_{2}}, \\
\left(1-\left(1-v_{2}^{\lambda q}\right)^{w_{2}}\right)+\left(1-\left(1-v_{2}^{\lambda q}\right)^{w_{2}}\right)-
\end{array}\right) \\
=\left\langle\left(\prod_{i=1}^{2}\left(\lambda \alpha_{i}\right)^{w_{i}}, \prod_{i=1}^{2}\left(\lambda \sigma_{i}\right)^{w_{i}}\right),\left(\prod_{i=1}^{2}\left(\left(1-\left(1-v_{i}^{q}\right)^{w_{2}}\right)\left(1-\left(1-v_{2}^{\lambda q}\right)^{w_{2}}\right)^{1 / q}\right)^{w_{i}},\left(1-\prod_{i=1}^{2}\left(1-v_{i}^{q \lambda}\right)^{w_{i}}\right)^{1 / q}\right)\right\rangle
\end{gathered}
$$

(2) Supposing $n=k, k>2$, that is

$$
\begin{gathered}
\prod_{i=1}^{k}\left(\lambda a_{i}\right)^{w_{i}}=\left(\lambda a_{1}\right)^{w_{1}} \otimes\left(\lambda a_{1}\right)^{w_{1}} \otimes \cdots \otimes\left(\lambda a_{k}\right)^{w_{k}} \\
=\left\langle\left(\prod_{i=1}^{k}\left(\lambda \alpha_{i}\right)^{w_{i}}, \prod_{i=1}^{k}\left(\lambda \sigma_{i}\right)^{w_{i}}\right),\right. \\
\left.\left(\prod_{i=1}^{k}\left(\left(1-\left(1-u_{i}^{q}\right)^{\lambda}\right)^{1 / q}\right)^{w_{i}},\left(1-\prod_{i=1}^{k}\left(1-v_{i}^{q \lambda}\right)^{w_{i}}\right)^{1 / q}\right)\right\rangle
\end{gathered}
$$

If $n=k+1$, according to the operational laws of q-RONFN, we can get

$$
\begin{gathered}
\prod_{i=1}^{k+1}\left(\lambda a_{i}\right)^{w_{i}}=\left(\lambda a_{1}\right)^{w_{1}} \otimes\left(\lambda a_{1}\right)^{w_{1}} \otimes \cdots \otimes\left(\lambda a_{k}\right)^{w_{k}} \otimes\left(\lambda a_{k+1}\right)^{w_{k+1}} \\
\left(\prod_{i=1}^{k}\left(\lambda \alpha_{i}\right)^{w_{i}}\left(\lambda \alpha_{k+1}\right)^{w_{k+1}}, \prod_{i=1}^{k}\left(\lambda \sigma_{i}\right)^{w_{i}}\left(\lambda \sigma_{k+1}\right)^{w_{k+1}}\right), \\
=\left\langle\left(\begin{array}{c}
\prod_{i=1}^{k}\left(\left(1-\left(1-u_{i}^{q}\right)^{\lambda}\right)^{1 / q}\right)^{w_{i}}\left(\left(1-\left(1-u_{k+1}^{q}\right)^{\lambda}\right)^{1 / q}\right)^{w_{k+1}}, \\
\left.\left(\begin{array}{c}
\left.\left(1-\prod_{i=1}^{k}\left(1-v_{i}^{q \lambda}\right)^{w_{i}}\right)^{1 / q}\right)^{q}+\left(\left(1-\prod_{i=1}^{k}\left(1-v_{k+1}^{q \lambda}\right)^{w_{k+1}}\right)^{1 / q}\right)^{q}- \\
\left(\left(1-\prod_{i=1}^{k}\left(1-v_{i}^{q \lambda}\right)^{w_{i}}\right)^{1 / q}\right)^{q}\left(\left(1-\prod_{i=1}^{k}\left(1-v_{k+1}^{q \lambda}\right)^{w_{k+1}}\right)^{1 / q}\right)^{q}
\end{array}\right)\right\rangle \\
\left(\prod_{i=1}^{k}\left(\lambda \alpha_{i}\right)^{w_{i}}\left(\lambda \alpha_{k+1}\right)^{w_{k+1}}, \prod_{i=1}^{k}\left(\lambda \sigma_{i}\right)^{w_{i}}\left(\lambda \sigma_{k+1}\right)^{w_{k+1}}\right), \\
\left(\begin{array}{l}
\prod_{i=1}^{k}\left(\left(1-\left(1-u_{i}^{q}\right)^{\lambda}\right)^{1 / q}\right)^{w_{i}}\left(\left(1-\left(1-u_{k+1}^{q}\right)^{\lambda}\right)^{1 / q}\right)^{w_{k+1}}, \\
\left(\begin{array}{l}
\left.1-\prod_{i=1}^{k}\left(1-v_{i}^{q \lambda}\right)^{w_{i}}+1-\prod_{i=1}^{k}\left(1-v_{k+1}^{q \lambda}\right)^{w_{k+1}}-\right)^{1 / q} \\
\left(1-\prod_{i=1}^{k}\left(1-v_{i}^{q \lambda}\right)^{w_{i}}\right)\left(1-\prod_{i=1}^{k}\left(1-v_{k+1}^{q \lambda}\right)^{w_{k+1}}\right)
\end{array}\right)
\end{array}\right) \\
=\left\langle\left(\prod_{i=1}^{k+1}\left(\lambda \alpha_{i}\right)^{w_{i}}, \prod_{i=1}^{k+1}\left(\lambda \sigma_{i}\right)^{w_{i}}\right),\left(\prod_{i=1}^{k+1}\left(\left(1-\left(1-u_{i}^{q}\right)^{\lambda}\right)^{1 / q}\right)^{w_{i}},\left(1-\prod_{i=1}^{k+1}\left(1-v_{i}^{q \lambda}\right)^{w_{i}}\right)^{1 / q}\right)\right.
\end{array}\right)\right.
\end{gathered}
$$


then

$$
\frac{1}{\lambda} \prod_{i=1}^{n}\left(\lambda a_{i}\right)^{w_{i}}=\left\langle\left(\frac{1}{\lambda} \prod_{i=1}^{n}\left(\lambda \alpha_{i}\right)^{w_{i}}, \frac{1}{\lambda} \prod_{i=1}^{n}\left(\lambda \sigma_{i}\right)^{w_{i}}\right),\left(\begin{array}{c}
\left(1-\left(1-\left(\prod_{i=1}^{n}\left(\left(1-\left(1-u_{i}^{q}\right)^{\lambda}\right)^{1 / q}\right)^{w_{i}}\right)^{q}\right)^{1 / \lambda}\right)^{1 / q} \\
\left(\left(1-\prod_{i=1}^{n}\left(1-v_{i}^{q \lambda}\right)^{w_{i}}\right)^{1 / q}\right)^{1 / \lambda}
\end{array}\right)\right\rangle .
$$

If $\lambda=1$, the Gq-RONFWG operator is reduced to the q-RONFWG operator. The Gq-RONFWG operator has the properties of boundedness and idempotency, these properties can be exemplified similar to Theorems $2-4$.

\subsection{Generalized q-RONF Ordered Weighted Aggregation Operators}

As generalizations of the q-RONFOWA and q-RONFOWG operators, some generalized q-RONF ordered weighted aggregation operators are introduced in the following.

Definition 15. Let $a_{i}=\left\langle\left(\alpha_{i}, \sigma_{i}\right),\left(u_{i}, v_{i}\right)\right\rangle(i=1,2, \cdots, n)$ be a collection of the $q$-RONFN, and $w_{j}=$ $\left(w_{1}, w_{2}, \cdots, w_{n}\right)^{T}$ be the weight vector of aggregation-associated, and $0 \leq w_{i} \leq 1(i=1,2, \cdots, n)^{T}, \sum_{i=1}^{n} w_{i}=$ $1, a_{\theta(i)}=\left\langle\left(\alpha_{\theta(i)}, \sigma_{\theta(i)}\right),\left(u_{\theta(i)}, v_{\theta(i)}\right)\right\rangle(i=1,2, \cdots, n)$ be the ith largest of them, then

(1) A generalized q-RONFN ordered weighted aggregation (Gq-RONFOWA) operator is a mapping Gq-RONFOWA: $a^{n} \rightarrow a$, where

$$
\begin{gathered}
\operatorname{Gq}-\operatorname{RONFOWA}\left(a_{1}, a_{2}, \cdots, a_{n}\right)=\left(\sum_{i=1}^{n} w_{i} a_{\theta(i)}^{\lambda}\right)^{1 / \lambda} \\
=\left\langle\left(\left(\sum_{i=1}^{n} w_{i} \alpha_{\theta(i)}^{\lambda}\right)^{1 / \lambda},\left(\sum_{i=1}^{n} w_{i} \sigma_{\theta(i)}^{\lambda}\right)^{1 / \lambda}\right),\right. \\
\left.\left.=\left\langle\left(1-\prod_{i=1}^{n}\left(1-u_{\theta(i)}^{q \lambda}\right)^{w_{i}}\right)^{1 / q}\right)^{1 / \lambda},\left(1-\left(1-\left(\prod_{i=1}^{n}\left(\left(1-\left(1-v_{\theta(i)}^{q}\right)^{\lambda}\right)^{1 / q}\right)^{w_{i}}\right)^{q}\right)^{1 / \lambda}\right)^{1 / q}\right)\right\rangle
\end{gathered}
$$

(2) A generalized q-RONFOWG (Gq-RONFOWG) operator is a mapping Gq-RONFOWG: $a^{n} \rightarrow a$, where

$$
=\left\langle\left(\frac{1}{\lambda} \prod_{i=1}^{n}\left(\lambda \alpha_{\theta(i)}\right)^{w_{i}}, \frac{1}{\lambda} \prod_{i=1}^{n}\left(\lambda \sigma_{\theta(i)}\right)^{w_{i}}\right),\left(\begin{array}{c}
\left(1-\left(1-\left(\prod_{i=1}^{n}\left(\left(1-\left(1-u_{\theta(i)}^{q}\right)^{\lambda}\right)^{1 / q}\right)^{w_{i}}\right)^{q}\right)^{1 / \lambda}\right. \\
\left(\left(1-\prod_{i=1}^{n}\left(1-v_{\theta(i)}^{q \lambda}\right)^{w_{i}}\right)^{1 / q}\right)^{1 / \lambda}
\end{array}\right)\right\rangle
$$

In the Equations (15) and (16), if $\lambda=1$, the Gq-RONFOWA and Gq-RONFOWG operators are reduced to the q-RONFOWA operator q-RONFOWG. According to Theorems $2-4$. We can similarly prove the properties of idempotency and boundedness and commutativity of q-RONFOWA operator q-RONFOWG operators.

\section{5. q-RONF Hybrid Aggregation Operators}

q-RONFWA, q-RONFWG, Gq-RONFWA and Gq-RONFWG operators only consider the importance of q-RONFN argument themselves, but they neglect the influence of the ordered position 
of the argument on the decision result. By contrast, q-RONFOWA, q-RONFOWG, GRONFOWA, and Gq-RONFOWG operators only pay attention to the ordered position of every q-RONFN argument, but they have no regard for the importance of the argument themselves. To solve the problems of operators above, we need to develop some hybrid aggregation operators to aggregate the q-RONFN information, which simultaneously consider both the arguments and ordered positions.

Definition 16. Let $a_{i}=\left\langle\left(\alpha_{i}, \sigma_{i}\right),\left(u_{i}, v_{i}\right)\right\rangle(i=1,2, \cdots, n)$ be a collection of the $q$-RONFN, $n$ is the balancing coefficient, and $w_{j}=\left(w_{1}, w_{2}, \cdots, w_{n}\right)^{T}$ be the weight vector of aggregation-associated, and $0 \leq$ $w_{i} \leq 1(i=1,2, \cdots, n)^{T}, \sum_{i=1}^{n} w_{i}=1, \omega_{j}=\left(\omega_{1}, \omega_{2}, \cdots, \omega_{n}\right)^{T}$ be the location weight vector, with $0 \leq \omega_{i} \leq$ $1(i=1,2, \cdots, n)^{T}, \sum_{i=1}^{n} \omega_{i}=1$.

(1) A q-RONF hybrid averaging ( $q$-RONFHA) operator is a mapping $q$-RONFHA: $a^{n} \rightarrow a$, where

$$
\begin{gathered}
\mathrm{q}-\operatorname{RONFHA}\left(a_{1}, a_{2}, \cdots, a_{n}\right)=\sum_{i=1}^{n} \omega_{i} \hat{a}_{\theta(i)} \\
=\left\langle\left(\sum_{i=1}^{n} \omega_{i} \hat{\alpha}_{\theta(i)}, \sum_{i=1}^{n} \omega_{i} \hat{\sigma}_{\theta(i)}\right),\left(\left(1-\prod_{i=1}^{n}\left(1-\hat{u}_{\theta(i)}^{q}\right)^{\omega_{i}}\right)^{1 / q}, \prod_{i=1}^{n} \hat{v}_{\theta(i)}^{\omega_{i}}\right)\right\rangle
\end{gathered}
$$

(2) A q-RONF hybrid geometric ( $q$-RONFHG) operator is a mapping $q$-RONFHG: $a^{n} \rightarrow a$, where

$$
\begin{gathered}
\mathrm{q}-\operatorname{RONFHG}\left(a_{1}, a_{2}, \cdots, a_{n}\right)=\prod_{i=1}^{n} \hat{\hat{a}}_{\theta(i)}^{\omega_{i}} \\
=\left\langle\left(\prod_{i=1}^{n} \hat{\hat{\alpha}}_{\theta(i)}^{\omega_{i}}, \prod_{i=1}^{n} \hat{\hat{\sigma}}_{\theta(i)}^{\omega_{i}}\right),\left(\prod_{i=1}^{n} \hat{\hat{u}}_{\theta(i)}^{\omega_{i}},\left(1-\prod_{i=1}^{n}\left(1-\hat{\hat{v}}_{\theta(i)}^{q}\right)^{\omega_{i}}\right)^{1 / q}\right)\right\rangle
\end{gathered}
$$

where $\hat{a}_{\theta(i)}$ is the ith largest of $q$-RONF $\hat{a}_{i}$, and $\hat{a}_{i}=n w_{i} a_{i}(i=1,2, \cdots, n), \hat{a}_{\theta(i)}$ is the ith largest of $q$-RONF $\hat{\hat{a}}_{i}$, and $\hat{\hat{a}}_{i}=a_{i}^{n w_{i}}(i=1,2, \cdots, n)$.

Definition 17. Let $a_{i}=\left\langle\left(\alpha_{i}, \sigma_{i}\right),\left(u_{i}, v_{i}\right)\right\rangle(i=1,2, \cdots, n)$ be a collection of the $q$-RONFN, $n$ is the balance coefficient, $\lambda$ be a parameter and $\lambda>0, w_{j}=\left(w_{1}, w_{2}, \cdots, w_{n}\right)^{T}$ be the weight vector of aggregation-associated, and $0 \leq w_{i} \leq 1(i=1,2, \cdots, n)^{T}, \sum_{i=1}^{n} w_{i}=1, \omega_{j}=\left(\omega_{1}, \omega_{2}, \cdots, \omega_{n}\right)^{T}$ be the location weight vector, with $0 \leq \omega_{i} \leq 1(i=1,2, \cdots, n)^{T}, \sum_{i=1}^{n} \omega_{i}=1$.

(1) A generalizations $q$-RONF hybrid averaging ( $G q$-RONFHA) operator is a mapping $G q$-RONFHA: $a^{n} \rightarrow a$, where

$$
\begin{gathered}
\operatorname{Gq}-\operatorname{RONFHA}\left(a_{1}, a_{2}, \cdots, a_{n}\right)=\left(\sum_{i=1}^{n} \omega_{i} \hat{a}_{\theta(i)}^{\lambda}\right)^{1 / \lambda} \\
=\left\langle\left(\left(\sum_{i=1}^{n} \omega_{i} \hat{\alpha}_{\theta(i)}^{\lambda}\right)^{1 / \lambda},\left(\sum_{i=1}^{n} \omega_{i} \hat{\sigma}_{\theta(i)}^{\lambda}\right)^{1 / \lambda}\right)\right. \\
\left.=\left\langle\left(\left(1-\prod_{i=1}^{n}\left(1-\hat{u}_{\theta(i)}^{q \lambda}\right)^{\omega_{i}}\right)^{1 / q}\right)^{1 / \lambda},\left(1-\left(1-\left(\prod_{i=1}^{n}\left(\left(1-\left(1-\hat{v}_{\theta(i)}^{q}\right)^{\lambda}\right)^{1 / q}\right)^{\omega_{i}}\right)^{q}\right)^{1 / \lambda}\right)^{1 / q}\right)\right\rangle
\end{gathered}
$$


(2) A generalizations $q$-RONF hybrid geometric (Gq-RONFHG) operator is a mapping Gq-RONFHG: $a^{n} \rightarrow a$, where

$$
=\left\langle\left(\frac{1}{\lambda} \prod_{i=1}^{n}\left(\lambda \hat{\hat{\alpha}}_{\theta(i)}\right)^{w_{i}}, \frac{1}{\lambda} \prod_{i=1}^{n}\left(\lambda \hat{\hat{\sigma}}_{\theta(i)}\right)^{w_{i}}\right),\left(\begin{array}{c}
\left(1-\left(1-\left(\prod_{i=1}^{n}\left(\left(1-\left(1-\hat{\hat{u}}_{\theta(i)}^{q}\right)^{\lambda}\right)^{1 / q}\right)^{w_{i}}\right)^{q}\right)^{1 / \lambda}\right)^{1 / q} \\
\left(\left(1-\prod_{i=1}^{n}\left(1-\hat{\hat{v}}_{\theta(i)}^{q \lambda}\right)^{w_{i}}\right)^{1 / q}\right)^{1 / \lambda}
\end{array}\right)\right)
$$

where $\hat{a}_{\theta(i)}$ is the ith lagest of $q$-RONF $\hat{a}_{i}$, and $\hat{a}_{i}=n w_{i} a_{i}(i=1,2, \cdots, n), \hat{a}_{\theta(i)}$ is the $i$-th largest of $q$-RONF $\hat{\hat{a}}_{i}$, and $\hat{\hat{a}}_{i}=a_{i}^{n n w_{i}}(i=1,2, \cdots, n)$.

\section{A Multi-Criteria Decision-Making Method Based on Q-rung Orthopair Normal Fuzzy Information}

For a multi-attribute decision making (MADM) problem under q-RONF environment, let $A=$ $\left\{A_{1}, A_{2}, \cdots, A_{n}\right\}$ denote $n$ alternative schemes, $C=\left\{C_{1}, C_{2}, \cdots, C_{m}\right\}$ denote $\mathrm{m}$ attribute sets for every alternative, and the corresponding weight is $w=\left\{w_{1}, w_{2}, \cdots, w_{m}\right\}$. The q-RONFN of scheme $A_{i}$ under attribute $C_{j}$ is $a_{i j}=\left\langle\left(\alpha_{i j}, \sigma_{i j}\right),\left(u_{i j}, v_{i j}\right)\right\rangle(i=1,2, \cdots, n ; j=1,2, \cdots, m)$. Where, $u_{i j}$ is the degree to which scheme $A_{i}$ belongs to normal fuzzy numbers $\left(\alpha_{i j}, \sigma_{i j}\right)$ under attribute $C_{j} ; v_{i j}$ is the degree to which scheme $A_{i}$ does not belong to normal fuzzy numbers $\left(\alpha_{i j}, \sigma_{i j}\right)$ under attribute $C_{j}, 0 \leq u_{i j}, v_{i j} \leq 1$, $0 \leq\left(u_{i j}\right)^{q}+\left(v_{i j}\right)^{q} \leq 1,(q \geq 1)$, A $n^{*} m$ decision matrix $D=\left(a_{i j}\right)_{n \times m}$ is composed by the set of $\mathrm{n}$ alternatives and the set of $\mathrm{m}$ attributes, and the following are the steps of MADM under q-RONF information environment:

Step 1. Normalizing the decision matrix.

Considering the differences among of the attributes' dimensions, in order to eliminate the influence of different dimensions on decision results, it is necessary to normalize the decision matrix $D=\left(a_{i j}\right)_{n \times m}$ to $\bar{D}=\left(\bar{a}_{i j}\right)_{n \times m^{\prime}}$ in which $\bar{a}_{i j}=\left\langle\left(\bar{\alpha}_{i j}, \bar{\sigma}_{i j}\right),\left(\bar{u}_{i j}, \bar{v}_{i j}\right)\right\rangle$.

For benefit-oriented attributes [51]:

$$
\bar{\alpha}_{i j}=\frac{\alpha_{i j}}{\max _{i}\left(\alpha_{i j}\right)}, \bar{\sigma}_{i j}=\frac{\sigma_{i j}}{\max _{i}\left(\sigma_{i j}\right)} \cdot \frac{\sigma_{i j}}{\alpha_{i j}}, \bar{u}_{i j}=u_{i j}, \bar{v}_{i j}=v_{i j} .
$$

For cost-oriented attributes [51]:

$$
\bar{\alpha}_{i j}=\frac{\min _{i}\left(\alpha_{i j}\right)}{\alpha_{i j}}, \bar{\sigma}_{i j}=\frac{\sigma_{i j}}{\max _{i}\left(\sigma_{i j}\right)} \cdot \frac{\sigma_{i j}}{\alpha_{i j}}, \bar{u}_{i j}=u_{i j}, \bar{v}_{i j}=v_{i j} .
$$

Step 2. Aggregating scheme attribute values

The information sets $\bar{a}_{i j}=\left\langle\left(\bar{\alpha}_{i j}, \bar{\sigma}_{i j}\right),\left(\bar{u}_{i j}, \bar{v}_{i j}\right)\right\rangle$ of attribute $C_{j}$ in scheme $A_{i}$ are aggregated into $\bar{a}_{i}=\left\langle\left(\bar{\alpha}_{i}, \bar{\sigma}_{i}\right),\left(\bar{u}_{i}, \bar{v}_{i}\right)\right\rangle$ by using q-RONFWA operator; the following results can be obtained

$$
\bar{a}_{i}=q-\operatorname{RONFWA}\left(\bar{a}_{i 1}, \bar{a}_{i 2}, \cdots, \bar{a}_{i m}\right)
$$

Step 3. Calculating score value $S\left(\bar{a}_{i}\right)$ and accuracy value $H\left(\bar{a}_{i}\right)$ of $\bar{a}_{i}$ by using score function and accuracy function

Step 4. Sorting the alternatives based on the q-RONFN sorting rules and choosing the best one. 


\section{Numerical Example}

\subsection{Ranking All Alternatives to Get Decision Results}

As economic globalization makes enterprises face more complex internal and external environment, finding an appropriate partner is an important way to maintain their competitiveness, which is affected by many factors. In order to select a suitable global partner, an enterprise has selected five candidate enterprises in the global scope. The set of alternative enterprises is $A=\left\{A_{1}, A_{2}, A_{3}, A_{4}, A_{5}\right\}$, and four attributes are considered, namely, R\& D capability $\left(C_{1}\right)$, business operation level $\left(C_{2}\right)$, international cooperation level $\left(C_{3}\right)$ and credit level $\left(C_{4}\right)$. The set of attributes $C=\left\{C_{1}, C_{2}, C_{3}, C_{4}\right\}$ is formed, and they are all benefit-oriented indicators. The corresponding weight is $w=\{0.3,0.2,0.2,0.3\}^{T}$, and the decision information matrix as shown in Table 1 is constructed according to the decision information. In addition, considering the problem of q-RONF information aggregation based on OWA operator, the corresponding position weight vector is given as $\omega=\{0.2,0.3,0.3,0.2\}^{T}$.

Table 1. Initial decision matrix.

\begin{tabular}{ccccc}
\hline & $C_{1}$ & $C_{2}$ & $C_{3}$ & $C_{4}$ \\
\hline$A_{1}$ & $<(8,0.7),(0.6,0.7)>$ & $<(4,0.5),(0.2,0.4)>$ & $<(8,0.5),(0.1,0.7)>$ & $<(4,0.3),(0.6,0.2)>$ \\
$A_{2}$ & $<(6,0.4),(0.7,0.9)>$ & $<(5,0.6),(0.4,0.3)>$ & $<(4,0.4),(0.7,0.2)>$ & $<(8,0.5),(0.6,0.4)>$ \\
$A_{3}$ & $<(9,0.8),(0.5,0.4)>$ & $<(7,0.8),(0.7,0.2)>$ & $<(4,0.3),(0.8,0.8)>$ & $<(6,0.6),(0.3,0.5)>$ \\
$A_{4}$ & $<(7,0.6),(0.7,0.8)>$ & $<(8,0.7),(0.8,0.6)>$ & $<(5,0.4),(0.6,0.9)>$ & $<(6,0.4),(0.7,0.3)>$ \\
$A_{5}$ & $<(5,0.3),(0.8,0.4)>$ & $<(7,0.5),(0.5,0.5)>$ & $<(5,0.3),(0.6,0.6)>$ & $<(8,0.6),(0.8,0.7)>$ \\
\hline
\end{tabular}

Step 1 The initial decision matrix $D=\left(a_{i j}\right)_{5 \times 4}$ is normalized and the normalized matrix $\bar{D}=\left(\bar{a}_{i j}\right)_{5 \times 4}$ is obtained. The results are shown in Table 2 .

Table 2. Normalized decision matrix.

\begin{tabular}{ccccc}
\hline & $C_{1}$ & $C_{2}$ & $C_{3}$ & $C_{4}$ \\
\hline$A_{1}$ & $<(0.889,0.077),(0.6,0.7)>$ & $<(0.5,0.078),(0.2,0.4)>$ & $<(1,063),(0.1,0.7)>$ & $<(0.5,0.038),(0.6,0.2)>$ \\
$A_{2}$ & $<(0.667,0.033),(0.7,0.9)>$ & $<(0.625,0.09),(0.4,0.3)>$ & $<(0.5,0.08),(0.7,0.2)>$ & $<(1,0.052),(0.6,0.4)>$ \\
$A_{3}$ & $<(1,0.089),(0.5,0.4)>$ & $<(0.875,0.114),(0.7,0.2>$ & $<(0.5,0.045),(0.8,0.8)>$ & $<(0.75,0.1),(0.3,0.5)>$ \\
$A_{4}$ & $<(0.778,0.064),(0.7,0.8)>$ & $<(1,0.077),(0.8,0.6)>$ & $<(0.625,0.064),(0.6,0.9)>$ & $<(0.75,0.044),(0.7,0.3)>$ \\
$A_{5}$ & $<(0.556,0.023),(0.8,0.4)>$ & $<(0.875,0.045),(0.5,0.5>$ & $<(0.625,0.036),(0.6,0.6)>$ & $<(1,0.075),(0.8,0.7)>$ \\
\hline
\end{tabular}

Step 2 q-RONFWA operator is used to aggregate the information in Table 2 to get the comprehensive q-RONFs of each scheme.

$$
\begin{aligned}
& \bar{a}_{1}=<(0.717,0.062),(0.516,0.43)>; \bar{a}_{2}=<(0.725,0.06),(0.635,0.419)>; \\
& \bar{a}_{3}=<(0.8,0.089),(0.622,0.428)>; \bar{a}_{4}=<(0.783,0.061),(0.71,0.576)>; \\
& \bar{a}_{5}=<(0.767,0.045),(0.735,0.537)>
\end{aligned}
$$

Step 3 The sorting result of score values based on q-RONFWA operator is calculated, as shown in the following:

$$
S\left(A_{1}\right)=0.042 ; S\left(A_{2}\right)=0.132 ; S\left(A_{3}\right)=0.13 ; S\left(A_{4}\right)=0.131 ; S\left(A_{5}\right)=0.186
$$

Step 4 According to the score value of each scheme, 5 schemes can be ranked as $A_{5}>A_{2}>A_{4}>$ $A_{3}>A_{1}$, so the best one is $A_{5}$.

\subsection{Comparative Analysis}

In order to analyze the validity and rationality of the proposed method in this paper, the sorting results based on different operators were analyzed, and compared with some existing methods.

Based on the steps in Section 5, the other 11 q-RONF aggregation operators proposed in this paper are used to calculate the sorting results. The results are shown in Table 3, which shows that the optimal scheme is $A_{5}$ based on q-RONFWA and q-RONFWG operators, which only consider the importance of 
attribute information themselves. However, the sorting result is $A_{5}$ by q-RONFOWA and q-RONFOWG operators, which attach the importance of weighted priority by using q-RONFOWA and q-RONFOWG operators. That indicates that weighted priority affects the sorting result of alternatives. Further, the sorting result based on generalized operators was analyzed, and let $\lambda=2$. Table 3 shows that the optimal decision results based on Gq-RONFWA, Gq-RONFWG and Gq-RONFOWG operators are $A_{5}$, and the optimal scheme based on Gq-RONFOWA operator is $A_{3}$. In the generalization operators, the optimal schemes based on q-RONFHA, q-RONFHG, Gq-RONFHA operators are $A_{5}$, which indicates that the parameter $\lambda$ and priority position weight also affect the sorting result. In practical decision-making problems, the most appropriate information aggregation operator can be chosen according to the actual requirement to obtain the corresponding decision-making results.

Table 3. Schemes sorting results based on different aggregation operators.

\begin{tabular}{|c|c|c|c|c|c|c|}
\hline \multirow{2}{*}{$\begin{array}{c}\text { Aggregation } \\
\text { Operator }\end{array}$} & \multicolumn{5}{|c|}{ The Score Value of Alternative } & \multirow{2}{*}{$\begin{array}{l}\text { Alternative } \\
\text { Ranking }\end{array}$} \\
\hline & $A_{1}$ & $A_{2}$ & $A_{3}$ & $A_{4}$ & $A_{5}$ & \\
\hline q-RONFWA & 0.042 & 0.132 & 0.13 & 0.131 & 0.186 & $\begin{array}{c}A_{5}>A_{2}>A_{4}> \\
A_{3}>A_{1}\end{array}$ \\
\hline q-RONFWG & -0.112 & -0.09 & -0.045 & -0.058 & 0.094 & $\begin{array}{c}A_{5}>A_{3}>A_{4}> \\
A_{2}>A_{1}\end{array}$ \\
\hline q-RONFOWA & 0.0141 & 0.1291 & 0.1605 & 0.1381 & 0.151 & $\begin{array}{c}A_{3}>A_{5}>A_{4}> \\
A_{2}>A_{1}\end{array}$ \\
\hline q-RONFOWG & -0.127 & -0.052 & -0.046 & -0.06 & 0.0662 & $\begin{array}{c}A_{3}>A_{2}>A_{4}> \\
A_{5}>A_{1}\end{array}$ \\
\hline Gq-RONFWA & 0.0702 & 0.1557 & 0.1805 & 0.1568 & 0.2134 & $\begin{array}{c}A_{5}>A_{3}>A_{4}> \\
A_{2}>A_{1}\end{array}$ \\
\hline Gq-RONFWG & -0.144 & -0.171 & -0.104 & -0.107 & 0.0646 & $\begin{array}{c}A_{5}>A_{3}>A_{4}> \\
A_{1}>A_{2}\end{array}$ \\
\hline Gq-RONFOWA & 0.047 & 0.151 & 0.2066 & 0.1669 & 0.1766 & $\begin{array}{c}A_{3}>A_{5}>A_{4}> \\
A_{2}>A_{1}\end{array}$ \\
\hline Gq-RONFOWG & -1.58 & -1.443 & -1.094 & -1.109 & 0.4111 & $\begin{array}{c}A_{5}>A_{3}>A_{4}> \\
A_{2}>A_{1}\end{array}$ \\
\hline q-RONFHA & 0.106 & 0.218 & 0.259 & 0.291 & 0.359 & $\begin{array}{c}A_{5}>A_{4}>A_{3}> \\
A_{2}>A_{1}\end{array}$ \\
\hline q-RONFHG & -0.1483 & -0.1483 & -0.1172 & -0.164 & -0.0012 & $\begin{array}{c}A_{5}>A_{3}>A_{2}> \\
A_{1}>A_{4}\end{array}$ \\
\hline Gq-RONFHA & 0.163 & 0.266 & 0.3224 & 0.3223 & 0.42 & $\begin{array}{c}A_{5}>A_{3}>A_{4}> \\
A_{2}>A_{1}\end{array}$ \\
\hline Gq-RONFHG & -1.182 & -2.413 & -1.665 & -2.073 & -0.263 & $\begin{array}{c}A_{5}>A_{3}>A_{1}> \\
A_{4}>A_{2}\end{array}$ \\
\hline
\end{tabular}

Compared with the results calculated by the existing information aggregation operators based on intuitionistic normal fuzzy numbers, the normal intuitionistic fuzzy weighted Bonferroni mean operator (the parameters $p=q=2$ ) proposed by Liu and Liu [54] was applied to the q-RONF environment. The optimal result is the same as those based on q-RONFWA and q-RONFWG in this paper as $A_{5}$. The normal intuitionistic fuzzy Heronian mean averaging and arithmetic operators (the parameters $p=q=2$ ) proposed by Zhang et al. [58] were applied to q-RONF environment, and the optimal result is also $A_{5}$. The comparative analysis shows that the proposed method is validity.

\subsection{Sensitivity Analysis of Parameters $q$ and $\lambda$}

In this Section, the influence of parameters $q$ and $\lambda$ on sorting result was analyzed. Firstly, the influences of the change of parameter $q$ in q-RONFWA and q-RONFOWA on the sorting result are discussed. As shown in Figures 1 and 2, the sorting result based on q-RONFWA operator shows that when $q$ changes from 2 to 12 , the final sorting result is always maintained at $A_{5}>A_{2}>A_{4}>A_{3}>A_{1}$, and the optimal selection is always $A_{5}$. The sorting result based on q-RONFWA operator shows that when $q>3$, the sorting result is always maintained at $A_{5}>A_{3}>A_{4}>A_{2}>A_{1}$, and only when $q=3$, 
the sorting result is $A_{3}>A_{5}>A_{4}>A_{2}>A_{1}$, indicating that the change of $q$ has little influence on the sorting result, which shows that the proposed method has strong stability.

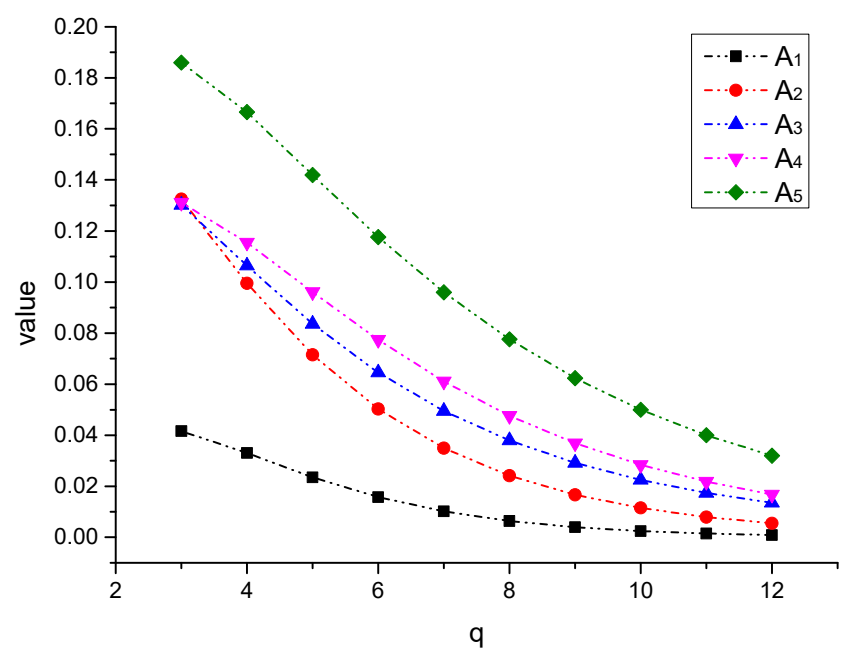

Figure 1. The influence of $q$-value change based on q-RONFWA operator on the sorting result.

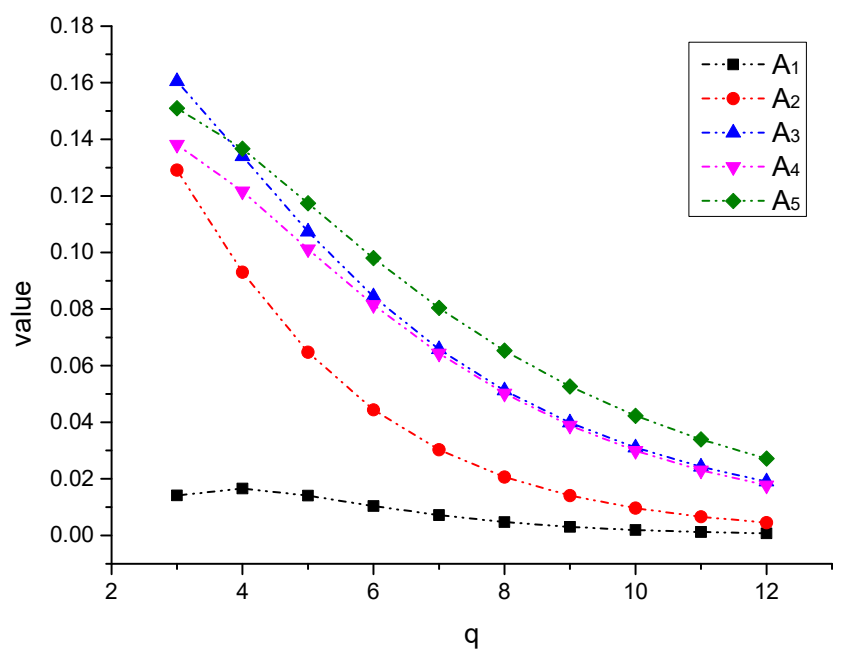

Figure 2. The influence of $q$-value change based on q-RONFOWA operator on the sorting result.

The influences of parameter $\lambda$ on the sorting result in generalized information aggregation operators are analyzed. As shown in Figures 3 and 4, based on the influence of parameter $\lambda$ in q-RONFWA operator on the sorting result, when $2 \leq \lambda \leq 5$, the sorting result is $A_{5}>A_{3}>A_{4}>A_{2}>A_{1}$, $A_{5}$ is the best choice. When $\lambda \geq 6$, the sorting result is $A_{3}>A_{5}>A_{4}>A_{2}>A_{1}$, and the optimal scheme is $A_{3}$. Based on the influence of parameter $\lambda$ in q-RONFOWA operator on the sorting result, when $2 \leq \lambda \leq 4$, the sorting result is $A_{3}>A_{5}>A_{4}>A_{2}>A_{1}$, and the optimal scheme is $A_{3}$. When $\lambda \geq 5, A_{3}>A_{4}>A_{5}>A_{2}>A_{1}$, although the optimal scheme remains unchanged, its ranking result changes. Thus, it is clear that the change of parameter $\lambda$ has a certain impact on the sorting result. Decision makers can adjust parameter $\lambda$ according to their subjective preferences and actual needs to obtain the desired results. 


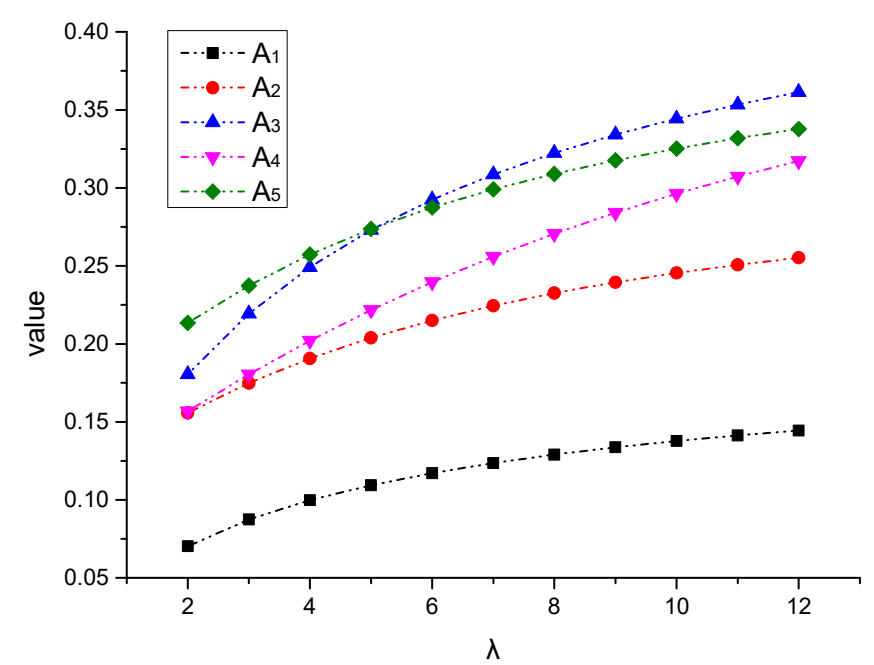

Figure 3. The influence of the change of $\lambda$ value based on Gq-RONFWA operator on the sorting result.

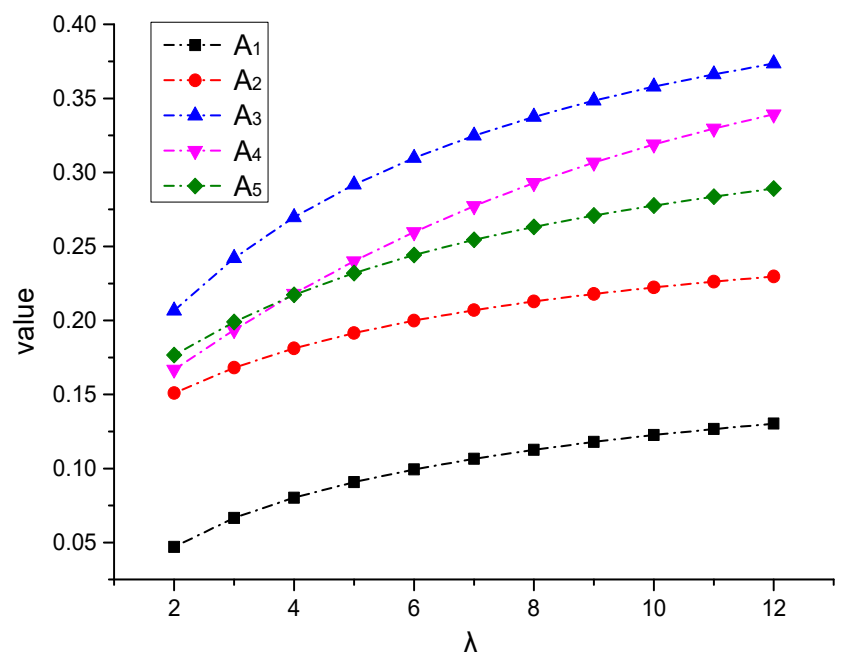

Figure 4. The influence of the change of $\lambda$ value based on Gq-RONFOWA operator on the sorting result.

The influence of the simultaneous changes of parameters $q$ and $\lambda$ on the ranking results was further analyzed, as shown in Figures 5 and 6. The influence of the change of $q$ and $\lambda$ in Gq-RONFHA operator on the sorting result shows that the total sorting is changed with the change of $q$ and $\lambda$. For example, when $q=3$ and $\lambda=2$, the sorting is $A_{5}>A_{3}>A_{4}>A_{2}>A_{1}$. If both $q$ and $\lambda$ increase at the same time, the sorting will change slightly, for example, when $q=13$ and $\lambda=12$, the sorting will be $A_{5}>A_{3}>A_{4}>A_{1}>A_{2}$. According to the change of $q$ and $\lambda$ in Gq-RONFHG operator, when $q=3$ and $\lambda=2$, then $A_{5}>A_{3}>A_{1}>A_{4}>A_{2}$; if both $q$ and $\lambda$ increase, for example when $q \geq 4$ and $\lambda \geq 3$, then $A_{5}>A_{1}>A_{3}>A_{4}>A_{2}$. It indicates that the simultaneous changes of parameters $\mathrm{q}$ and $\lambda$ have some influence on the ranking results. However, within a certain range where the two parameters change together, the optimal results remain unchanged, which shows that the method presented in this paper has certain stability. 


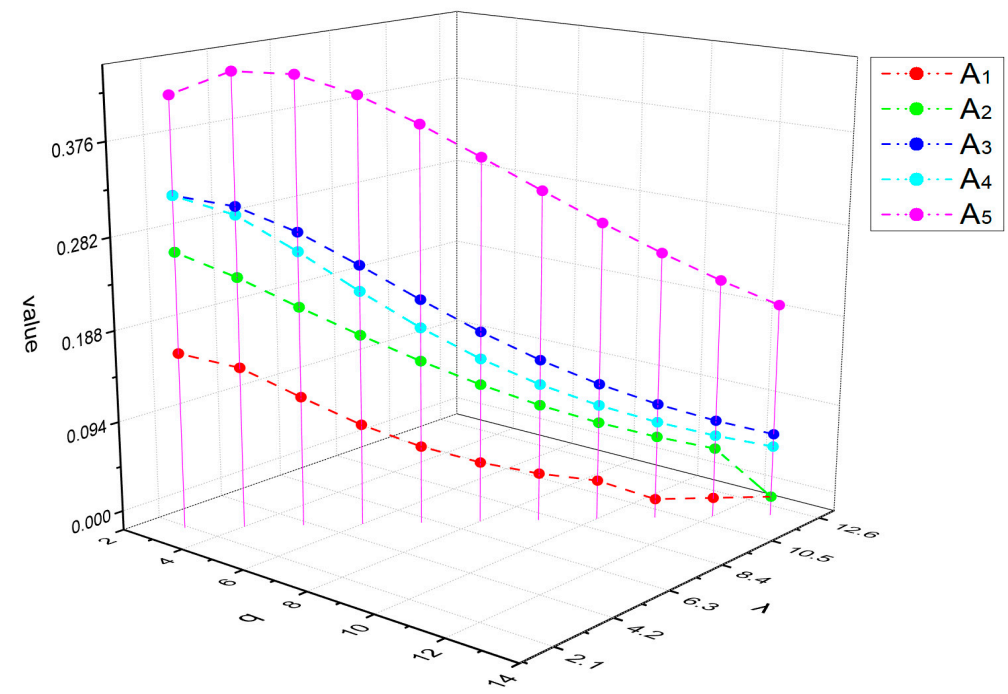

Figure 5. The influence of the simultaneous change of $q$ value and $\lambda$ in Gq-RONFHA operator on the sorting result.

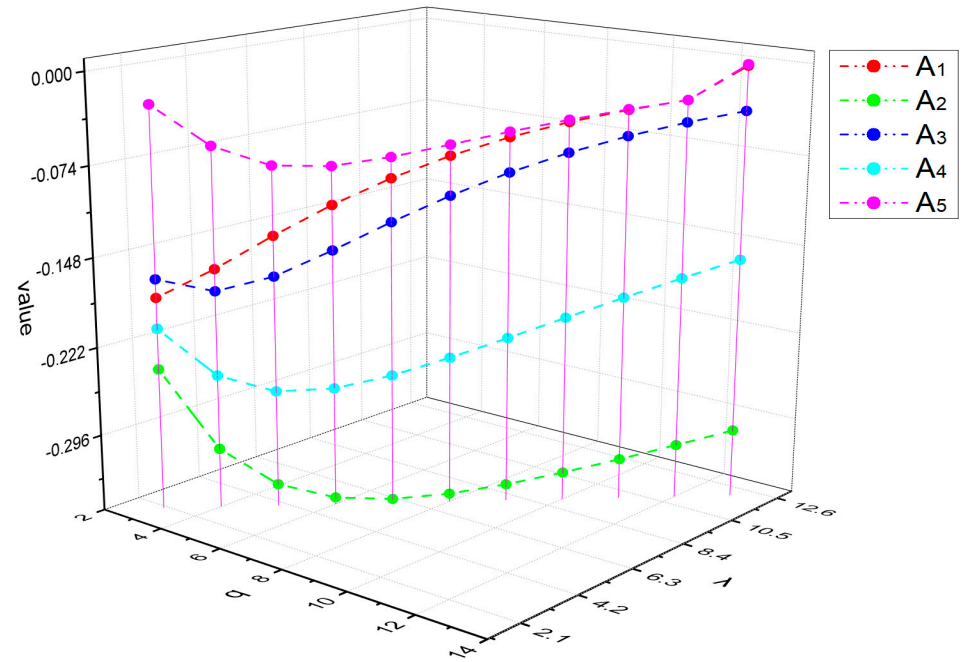

Figure 6. The influence of the simultaneous change of $q$ value and $\lambda$ in Gq-RONFHG operator on the sorting result.

\subsection{Discussion}

According to the above numerical example, we can easily find the proposed methods can depict the social and natural phenomena that obey normal distribution, and take into account that the sum of MED and NOMED is greater than 1, but the fuzzy information whose q power is less than 1. As such, our methods can analyze more complicated fuzzy information and objective phenomena; its decision process is closer to the way of human thinking. Based on the new concept of q-RONF set, we presented 12 aggregation operators under q-RONF environment; the research results showed that these operators have stronger information aggregation ability than existing operators. The proposed q-RONF set and the aggregation operators can set different $q$ values according to actual requirements to get more appropriate decision results. As a result, our method is more flexible and more suitable for decision-making in a more complex and changeable decision-making environment.

To sum up, the proposed method has stronger applicability and flexibility in solving MADM problems under uncertain environments, especially in solving decision-making problems that are difficult to deal with normal fuzzy information environment or q-RON information environment. 


\section{Conclusions}

The q-rung orthopair fuzzy set (q-ROFS) can characterize fuzzy information more effectively and extensively, and it has more flexibility than IFS and PFS, but it is difficult for q-ROFS to characterize normal distribution in human society and nature. To solve this problem, in this paper, and considering the characterization ability of normal fuzzy numbers and the description breadth of q-ROFS for fuzzy information. The paper proposes a new concept of q-rung orthopair normal fuzzy (q-RONF) set by combining normal fuzzy numbers and q-ROFS. In addition, some basic properties of q-RONF set are examined, some information aggregation operators were proposed and applied as well. The main contributions of this paper are as follows:

(1) The concept and the operation rules of q-RONF set were defined, their related properties of the operation rules were proved. Meanwhile, the score function and the accuracy function under the q-RONF information environment were defined.

(2) Some information aggregation operators in q-RONF environment was proposed, including q-RONF weighted averaging and geometric operators, q-RONF order weighted averaging and geometric operators, generalized q-RONF weighted averaging and geometric operators, generalized order weighted averaging, and geometric operators, q-RONF hybrid averaging and geometric operators, generalized q-RONF hybrid averaging and geometric operators. Further, some properties of the above aggregation operators are analyzed, such as commutativity, idempotency, and boundedness.

(3) A multi-attribute decision-making method based on q-RONF information aggregation operators was proposed.

In future work, the q-RONFS can be extended to interval q-RONFS, the Choquet and power integral operators can be applied into q-RONFS to develop new aggregation operators. Moreover, the q-RONFS can be widely used in practical decision making, such as the supply of chain management and system control.

Author Contributions: Conceptualization, Z.Y. and J.L.; methodology, X.L. and Z.C.; software, Z.Y. and X.L.; validation, Z.Y. and J.L.; data curation, X.L.; All the authors have participated in writing the manuscript.

Funding: This research was supported in part by the Natural Science Foundation of China (No. 71704007), and the Beijing Social Science Foundation of China (No. 18GLC082).

Conflicts of Interest: The authors declare no conflict of interest.

\section{References}

1. Zadeh, L.A. Fuzzy sets. Inform. Control. 1965, 8, 338-353. [CrossRef]

2. Atanassov, K.T. Intuitionistic fuzzy sets. Fuzzy Sets Syst. 1986, 20, 87-96. [CrossRef]

3. Atanassov, K.; Gargov, G. Interval valued Intuitionistic fuzzy-sets. Fuzzy Sets Syst. 1989, 31, $343-349$. [CrossRef]

4. Wan, S.P.; Xu, G.L.; Wang, F.; Dong, J.Y. A new method for Atanassov's interval-valued intuitionistic fuzzy MAGDM with incomplete attribute weight information. Inform. Sci. 2015, 316, 329-347. [CrossRef]

5. Liu, P.D.; Chen, S.M. Multiattribute group decision making based on intuitionistic 2-tuple linguistic information. Inform. Sci. 2018, 430, 599-619. [CrossRef]

6. Wang, J.Q.; Zhang, Z. Aggregation operators on intuitionistic trapezoidal fuzzy number and its application to multi-criteria decision making problems. J. Syst. Eng. Electron. 2009, 20, 321-326.

7. Nehi, H.M. A New Ranking Method for Intuitionistic Fuzzy Numbers. Int. J. Fuzzy. Syst. 2010, 12, 80-86.

8. Wang, J.Q.; Li, K.J.; Zhang, H.Y. Multi-criteria decision-making method based on induced intuitionistic normal fuzzy related aggregation operators. Int. J. Uncertain. Fuzzy. 2012, 20, 559-578. [CrossRef]

9. Liu, P.D.; Jin, F. Methods for aggregating intuitionistic uncertain linguistic variables and their application to group decision making. Inform. Sci. 2012, 205, 58-71. [CrossRef]

10. Li, D.F. A ratio ranking method of triangular intuitionistic fuzzy numbers and its application to MADM problems. Comput. Math. Appl. 2010, 60, 1557-1570. [CrossRef]

11. Li, D.F.; Nan, J.X.; Zhang, M.J. A Ranking Method of Triangular Intuitionistic Fuzzy Numbers and Application to Decision Making. Int. J. Comput. Int. Sys. 2010, 3, 522-530. [CrossRef] 
12. Yager, R.R. Pythagorean membership grades in multicriteria decision making. IEEE Trans. Fuzzy Syst. 2014, 22, 958-965. [CrossRef]

13. Yager, R.R.; Abbasov, A.M. Pythagorean membership grades, complex numbers, and decision making. Int. J. Intell. Syst. 2013, 28, 436-452. [CrossRef]

14. Akram, M.; Dar, J.M.; Farooq, A. Planar graphs under Pythagorean fuzzy environment. Mathematics 2018, 6, 278. [CrossRef]

15. Liang, D.C.; Xu, Z.S.; Liu, D.; Wu, Y. Method for three-way decisions using ideal TOPSIS solutions at Pythagorean fuzzy information. Inform. Sci. 2018, 435, 282-295. [CrossRef]

16. Deng, X.M.; Wang, J.; Wei, G.W.; Lu, M. Models for multiple attribute decision making with some 2-tuple linguistic Pythagorean fuzzy Hamy mean operators. Mathematics 2018, 6, 236. [CrossRef]

17. Liu, A.J.; Ji, X.H.; Lu, H.; Liu, H.Y. The selection of 3PRLs on self-service mobile recycling machine: Interval-valued pythagorean hesitant fuzzy best-worst multi-criteria group decision-making. J. Clean. Prod. 2019, 230, 734-750. [CrossRef]

18. Rahman, K.; Abdullah, S. Some induced generalized interval-valued Pythagorean fuzzy Einstein geometric aggregation operators and their application to group decision-making. Comput. Appl. Math. 2019, 38, 139. [CrossRef]

19. Tang, M.; Wang, J.; Lu, J.P.; Wei, G.W.; Wei, C.; Wei, Y. Dual hesitant Pythagorean fuzzy Heronian mean operators in multiple attribute decision making. Mathematics 2019, 7, 344. [CrossRef]

20. Zhang, X.L. Multicriteria Pythagorean fuzzy decision analysis: A hierarchical QUALIFLEX approach with the closeness index-based ranking methods. Inf. Sci. 2016, 330, 104-124. [CrossRef]

21. Ren, P.J.; Xu, Z.S.; Gou, X.J. Pythagorean fuzzy TODIM approach to multi-criteria decision making. Appl. Soft. Comput. 2016, 42, 246-259. [CrossRef]

22. Garg, H. A new generalized pythagorean fuzzy information aggregation using einstein operations and its application to decision making. Int. J. Intell. Syst. 2016, 31, 886-920. [CrossRef]

23. Zhang, R.T.; Wang, J.; Zhu, X.M.; Xia, M.M.; Yu, M. Some Generalized Pythagorean Fuzzy Bonferroni Mean Aggregation Operators with Their Application to Multiattribute Group Decision-Making. Complexity 2017, 2017, 16. [CrossRef]

24. Yager, R.R. Generalized Orthopair Fuzzy Sets. IEEE Trans. Fuzzy Syst. 2017, 25, 1222-1230. [CrossRef]

25. Gao, J.; Liang, Z.L.; Shang, J.; Xu, Z.S. Continuities, Derivatives, and Differentials of q-Rung Orthopair Fuzzy Functions. IEEE Trans. Fuzzy Syst. 2019, 27, 1687-1699. [CrossRef]

26. Peng, X.D.; Dai, J.G.; Garg, H. Exponential operation and aggregation operator for q-rung orthopair fuzzy set and their decision-making method with a new score function. Int. J. Intell. Syst. 2018, 33, 2255-2282. [CrossRef]

27. Du, W.S. Research on arithmetic operations over generalized orthopair fuzzy sets. Int. J. Intell. Syst. 2019, 34, 709-732. [CrossRef]

28. Peng, X.D.; Dai, J.G. Research on the assessment of classroom teaching quality with q-rung orthopair fuzzy information based on multiparametric similarity measure and combinative distance-based assessment. Int. J. Intell. Syst. 2019, 34, 1588-1630. [CrossRef]

29. Wang, P.; Wang, J.; Wei, G.W.; Wei, C. Similarity measures of q-rung orthopair fuzzy sets based on cosine function and their applications. Mathematics 2019, 7, 340. [CrossRef]

30. Liu, D.H.; Chen, X.H.; Peng, D. Some cosine similarity measures and distance measures between q-rung orthopair fuzzy sets. Int. J. Intell. Syst. 2019, 34, 1572-1587. [CrossRef]

31. Luqman, A.; Akram, M.; Al-Kenani, A.N. Q-Rung orthopair fuzzy hypergraphs with applications. Mathematics 2019, 7, 260. [CrossRef]

32. Xu, Y.; Shang, X.P.; Wang, J.; Wu, W.; Huang, H.Q. Some q-Rung Dual Hesitant Fuzzy Heronian Mean Operators with Their Application to Multiple Attribute Group Decision-Making. Symmetry 2018, 10, 472. [CrossRef]

33. Ju, Y.B.; Luo, C.; Ma, J.; Wang, A.H. A novel multiple-attribute group decision-making method based on q-rung orthopair fuzzy generalized power weighted aggregation operators. Int. J. Intell. Syst. 2019, 34, 2077-2103. [CrossRef]

34. Wang, H.H.; Ju, Y.B.; Liu, P.D. Multi-attribute group decision-making methods based on q-rung orthopair fuzzy linguistic sets. Int. J. Intell. Syst. 2019, 34, 1129-1157. [CrossRef] 
35. Wang, J.; Gao, H.; Wei, G.W.; Wei, Y. Methods for Multiple-Attribute Group Decision Making with q-Rung Interval-Valued Orthopair Fuzzy Information and Their Applications to the Selection of Green Suppliers. Symmetry 2019, 11, 56. [CrossRef]

36. Wang, J.; Wei, G.W.; Wei, C.; Wei, Y. Dual Hesitant q-Rung Orthopair Fuzzy Muirhead Mean Operators in Multiple Attribute Decision Making. IEEE Access. 2019, 7, 67139-67166. [CrossRef]

37. Chen, K.; Luo, Y.D. Generalized orthopair linguistic Muirhead mean operators and their application in multi-criteria decision making. J. Intell. Fuzzy Syst. 2019, 37, 797-809. [CrossRef]

38. Wang, J.; Wei, G.; Lu, J.; Alsaadi, F.E.; Hayat, T.; Wei, C.; Zhang, Y. Some q-rung orthopair fuzzy Hamy mean operators in multiple attribute decision-making and their application to enterprise resource planning systems selection. Int. J. Intell. Syst. 2019, 34, 2429-2458. [CrossRef]

39. Gao, H.X.; Ju, Y.B.; Zhang, W.K.; Ju, D.W. Multi-Attribute Decision-Making Method Based on Interval-Valued q-Rung Orthopair Fuzzy Archimedean Muirhead Mean Operators. IEEE Access. 2019, 7, 74300-74315. [CrossRef]

40. Liu, P.D.; Liu, J.L. Some q-Rung Orthopai Fuzzy Bonferroni Mean Operators and Their Application to Multi-Attribute Group Decision Making. Int. J. Intell. Syst. 2018, 33, 315-347. [CrossRef]

41. Liu, Z.M.; Wang, S.; Liu, P.D. Multiple attribute group decision making based on q-rung orthopair fuzzy Heronian mean operators. Int. J. Intell. Syst. 2018, 33, 2341-2363. [CrossRef]

42. Liu, P.D.; Wang, P. Multiple-Attribute Decision-Making Based on Archimedean Bonferroni Operators of q-Rung Orthopair Fuzzy Numbers. IEEE Trans. Fuzzy Syst. 2019, 27, 834-848. [CrossRef]

43. Yang, W.; Pang, Y.F. New q-rung orthopair fuzzy partitioned Bonferroni mean operators and their application in multiple attribute decision making. Int. J. Intell. Syst. 2019, 34, 439-476. [CrossRef]

44. Xing, Y.P.; Zhang, R.T.; Zhu, X.M.; Bai, K.Y. Q-Rung orthopair fuzzy uncertain linguistic choquet integral operators and their application to multi-attribute decision making. J. Intell. Fuzzy Syst. 2019, 37, 1123-1139. [CrossRef]

45. Zhang, C.; Liao, H.C.; Luo, L. Additive consistency-based priority-generating method of q-rung orthopair fuzzy preference relation. Int. J. Intell. Syst. 2019, 34, 2151-2176. [CrossRef]

46. Peng, X.D.; Krishankumar, R.; Ravichandran, K.S. Generalized orthopair fuzzy weighted distance-based approximation (WDBA) algorithm in emergency decision-making. Int. J. Intell. Syst. 2019, 34, 2364-2402. [CrossRef]

47. Liu, D.H.; Peng, D.; Liu, Z.M. The distance measures between q-rung orthopair hesitant fuzzy sets and their application in multiple criteria decision making. Int. J. Intell. Syst. 2019, 34, 2104-2121. [CrossRef]

48. Hussain, A.; Ali, M.I.; Mahmood, T. Covering based q-rung orthopair fuzzy rough set model hybrid with TOPSIS for multi-attribute decision making. J. Intell. Fuzzy Syst. 2019, 37, 981-993. [CrossRef]

49. Yang, M.S.; Ko, C.H. On a class of fuzzy c-numbers clustering procedures for fuzzy data. Fuzzy Sets Syst. 1996, 84, 49-60. [CrossRef]

50. Li, D.Y.; Liu, C.Y. Study on the universality of the normal cloud model. Eng. Sci. 2004, 6, 28-34.

51. Wang, J.Q.; Li, K.J.; Zhang, H.Y.; Chen, X.H. A score function based on relative entropy and its application in intuitionistic normal fuzzy multiple criteria decision making. J. Intell. Fuzzy Syst. 2013, 25, 567-576.

52. Liu, P.D.; Teng, F. Multiple Criteria Decision Making Method based on Normal Interval-Valued Intuitionistic Fuzzy Generalized Aggregation Operator. Complexity 2016, 21, 277-290. [CrossRef]

53. Wang, J.Q.; Zhou, P.; Li, K.J.; Zhang, H.Y.; Chen, X.H. Multi-criteria decision-making method based on normal intuitionistic fuzzy-induced generalized aggregation operator. Top 2014, 22, 1103-1122. [CrossRef]

54. Liu, Z.M.; Liu, P.D. Normal intuitionistic fuzzy Bonferroni mean operators and their applications to multiple attribute group decision making. J. Intell. Fuzzy Syst. 2015, 29, 2205-2216. [CrossRef]

55. Yang, Z.L.; Li, J.Q.; Huang, L.C.; Shi, Y.Y. Developing dynamic intuitionistic normal fuzzy aggregation operators for multi-attribute decision-making with time sequence preference. Expert Syst. Appl. 2017, 82, 344-356. [CrossRef]

56. Li, J.Q.; Chen, W.; Yang, Z.L.; Li, C.Y.; Sellers, J.S. Dynamic interval-valued intuitionistic normal fuzzy aggregation operators and their applications to multi-attribute decision-making. J. Intell. Fuzzy Syst. 2018, 35, 3937-3954. [CrossRef]

57. Liu, P.D. Multiple Attribute Decision-Making Methods Based on Normal Intuitionistic Fuzzy Interaction Aggregation Operators. Symmetry 2017, 9, 261. [CrossRef] 
58. Zhang, G.F.; Zhang, Z.M.; Kong, H. Some Normal Intuitionistic Fuzzy Heronian Mean Operators Using Hamacher Operation and Their Application. Symmetry 2018, 10, 199. [CrossRef]

59. Xu, R.N.; Li, C.L. Regression prediction for fuzzy time series. Appl. Math. A. J. Chin. Uni. 2001, 16, 451-461.

60. Liu, P.D.; Wang, P. Some q-rung orthopair fuzzy aggregation operators and their applications to multiple-attribute decision making. Int. J. Intell. Syst. 2018, 33, 259-280. [CrossRef]

61. Xu, Z. Intuitionistic fuzzy aggregation operators. IEEE Trans. Fuzzy Syst. 2007, 15, 1179-1187.

62. Xu, Z. Dependent uncertain ordered weighted aggregation operators. Inform. Fusion 2008, 9, 310-316. [CrossRef]

(C) 2019 by the authors. Licensee MDPI, Basel, Switzerland. This article is an open access article distributed under the terms and conditions of the Creative Commons Attribution (CC BY) license (http://creativecommons.org/licenses/by/4.0/). 